\title{
Homological mirror symmetry for Milnor fibers of simple singularities
}

\author{
Yankı Lekili and Kazushi Ueda
}

\begin{abstract}
We prove homological mirror symmetry for Milnor fibers of simple singularities in dimensions greater than 1, which are among the log Fano cases of Conjecture 1.5 of the authors' manuscript "Homological mirror symmetry for Milnor fibers via moduli of $A_{\infty}$-structures" [arXiv: 1806.04345]. The proof is based on a relation between matrix factorizations and Calabi-Yau completions. As an application, we give an explicit computation of the Hochschild cohomology group of the derived $n$-preprojective algebra of a Dynkin quiver for any $n \geqslant 1$, and the symplectic cohomology group of the Milnor fiber of any simple singularity in any dimension greater than 1 .
\end{abstract}

\section{Introduction}

A simple singularity is an isolated hypersurface singularity of modality 0. Arnold classified such singularities; up to right equivalence, they are given by one of the following:

$$
\begin{aligned}
& A_{\ell}: x_{1}^{\ell+1}+x_{2}^{2}+\cdots+x_{n+1}^{2}=0, \quad \ell=1,2, \ldots, \\
& D_{\ell}: x_{1}^{\ell-1}+x_{1} x_{2}^{2}+x_{3}^{2}+\cdots+x_{n+1}^{2}=0, \quad \ell=4,5, \ldots, \\
& E_{6}: x_{1}^{4}+x_{2}^{3}+x_{3}^{2}+\cdots+x_{n+1}^{2}=0, \\
& E_{7}: x_{1}^{3}+x_{1} x_{2}^{3}+x_{3}^{2}+\cdots+x_{n+1}^{2}=0, \\
& E_{8}: x_{1}^{5}+x_{2}^{3}+x_{3}^{2}+\cdots+x_{n+1}^{2}=0 .
\end{aligned}
$$

In the case $n=2$, simple surface singularities have many other characterizations, such as Kleinian singularities, rational double points, or canonical singularities, to name a few.

Let $\check{w}$ be one of these defining polynomials, which we think of as a holomorphic function on $\mathbb{C}^{n+1}$, and equip $\check{w}^{-1}(1)$ with the Liouville structure induced from the standard one on $\mathbb{C}^{n+1}$. This is the Liouville completion of the Milnor fiber, which is the Liouville domain obtained by intersecting $\check{w}^{-1}(1)$ with a ball. Let $\mathcal{W}\left(\check{w}^{-1}(1)\right)$ denote the idempotent-complete derived wrapped Fukaya category of $\check{w}^{-1}(1)$.

For $n \geqslant 2$, since $\check{w}^{-1}(1)$ is not a log Calabi-Yau manifold but a log Fano manifold, its mirror is not a manifold but a Landau-Ginzburg model, by which we mean a pair of a stack and a section

Received 1 May 2020, accepted in final form 11 February 2021.

2020 Mathematics Subject Classification 53D37.

Keywords: homologial mirror symmetry, simple singularity, symplectic cohomology.

This journal is (C) Foundation Compositio Mathematica 2021. This article is distributed with Open Access under the terms of the Creative Commons Attribution Non-Commercial License, which permits non-commercial reuse, distribution, and reproduction in any medium, provided that the original work is properly cited. For commercial re-use, please contact the Foundation Compositio Mathematica.

Y. L. is partially supported by the Royal Society URF $\backslash R \backslash 180024$. K. U. is partially supported by Grant-in-Aid for Scientific Research (15KT0105, 16K13743, 16H03930). 


\section{SiMPLE SINGULARITIES}

of a line bundle on it. One way to obtain a Landau-Ginzburg mirror of a log Fano manifold is to first remove a divisor to make it log Calabi-Yau, then find its mirror, which is another log Calabi-Yau manifold, and finally add a potential to this mirror [Aur07, Aur09]. This produces a Landau-Ginzburg mirror whose underlying manifold is of the same dimension as the original manifold. When the singularity is toric (that is, a simple surface singularity of type A), there is a standard choice for the divisor to remove, and the resulting mirror is the Landau-Ginzburg model consisting of a complement of a toric divisor in the minimal resolution of the singularity of the same type and a monomial function on it (see, for example, [AAK16, Section 9.2]). The choice of the divisor is not unique in general, and there are multiple mirrors for a given Milnor fiber.

In this paper, we consider an alternative mirror of the Milnor fiber of a simple singularity based on the transposition of invertible polynomials introduced in [BH93, BH95]. A weighted homogeneous polynomial $\mathbf{w} \in \mathbb{C}\left[x_{1}, \ldots, x_{n+1}\right]$ with an isolated critical point at the origin is invertible if there is an integer matrix $A=\left(a_{i j}\right)_{i, j=1}^{n+1}$ with non-zero determinant such that

$$
\mathbf{w}=\sum_{i=1}^{n+1} \prod_{j=1}^{n+1} x_{j}^{a_{i j}} .
$$

The transpose of $\mathbf{w}$ is defined as

$$
\check{w}=\sum_{i=1}^{n+1} \prod_{j=1}^{n+1} x_{j}^{a_{j i}},
$$

whose exponent matrix $\check{A}$ is the transpose matrix of $A$. The group

$$
\Gamma_{\mathbf{w}}:=\left\{\left(t_{0}, t_{1}, \ldots, t_{n+1}\right) \in\left(\mathbb{G}_{\mathrm{m}}\right)^{n+2} \mid t_{1}^{a_{1,1}} \cdots t_{n+1}^{a_{1, n+1}}=\cdots=t_{1}^{a_{n+1,1}} \cdots t_{n+1}^{a_{n+1, n+1}}=t_{0} t_{1} \cdots t_{n+1}\right\}
$$

acts naturally on $\mathbb{A}^{n+2}:=\operatorname{Spec} \mathbb{C}\left[x_{0}, \ldots, x_{n+1}\right]$. Let $\operatorname{mf}\left(\mathbb{A}^{n+2}, \Gamma_{\mathbf{w}}, \mathbf{w}+x_{0} \cdots x_{n+1}\right)$ denote the idempotent completion of the $\mathrm{dg}$ category of $\Gamma_{\mathbf{w}}$-equivariant coherent matrix factorizations of $\mathbf{w}+x_{0} \cdots x_{n+1}$ on $\mathbb{A}^{n+2}$ in the sense of [EP15]. Conjecture 1.1 below is given in [LU18, Conjecture 1.5].

Conjecture 1.1. For any invertible polynomial w, one has a quasi-equivalence

$$
\operatorname{mf}\left(\mathbb{A}^{n+2}, \Gamma_{\mathbf{w}}, \mathbf{w}+x_{0} \cdots x_{n+1}\right) \simeq \mathcal{W}\left(\check{w}^{-1}(1)\right) .
$$

In other words, the Landau-Ginzburg model $\left(\left[\mathbb{A}^{n+2} / \Gamma_{\mathbf{w}}\right], \mathbf{w}+x_{0} \cdots x_{n+1}\right)$ is mirror to the Liouville manifold $\check{w}^{-1}(1)$. The main result of this paper is the following.

Theorem 1.2. Conjecture 1.1 holds for $n \geqslant 2$ and $\check{w}$ one of the defining polynomials of simple singularities appearing in (1.1).

The proof of Theorem 1.2 consists of four steps. The first step is the quasi-equivalence

$$
\operatorname{mf}\left(\mathbb{A}^{n+2}, \Gamma_{\mathbf{w}}, \mathbf{w}+x_{0} \cdots x_{n+1}\right) \simeq \operatorname{mf}\left(\mathbb{A}^{n+2}, \Gamma_{\mathbf{w}}, \mathbf{w}\right),
$$

which comes from the fact that $\mathbf{w}+x_{0} \cdots x_{n+1}$ is right equivalent to $\mathbf{w}$ by a formal change of variables, which holds if $n \geqslant 2$ and $\mathbf{w}$ defines a simple singularity.

The second step is the quasi-equivalence

$$
\operatorname{mf}\left(\mathbb{A}^{n+2}, \Gamma_{\mathbf{w}}, \mathbf{w}\right) \simeq \Pi_{n}\left(\operatorname{mf}\left(\mathbb{A}^{n+1}, \Gamma_{\mathbf{w}}, \mathbf{w}\right)\right),
$$

where $\Pi_{n}$ denotes the $n$-Calabi-Yau completion in the sense of [Kel11]. This holds for any invertible polynomial $\mathbf{w}$ and any $n \geqslant 0$. 


\section{Y. LEKILI AND K. UEDA}

The third step is the quasi-equivalence

$$
\operatorname{mf}\left(\mathbb{A}^{n+1}, \Gamma_{\mathbf{w}}, \mathbf{w}\right) \simeq \operatorname{perf} A_{Q}
$$

with the $\operatorname{dg}$ category perf $A_{Q}$ of perfect $\operatorname{dg}$ modules over the path algebra $A_{Q}$ of a Dynkin quiver $Q$ (with any orientation) of the corresponding type. For type A, this is proved in [Tak05, Theorem 3.1] for $n=0$, and the $n \geqslant 1$ case follows either from the $n=0$ case and the Knörrer periodicity [Knö87], or as a special case of [FU11, Theorem 1.2]. For type D, this follows from [FU13, Section 4]. For type E, this follows either by combining [HS20, Theorem 1] and [Sei01, Proposition 3.4] or by finding a suitable mutation of a generator appearing in [HS20, Theorem 2]. Note that [KST07, Theorem 3.1] gives a result close to (1.4), which is not exactly the same since the grading group is different.

The last step is

$$
\mathcal{W}\left(\check{w}^{-1}(1)\right) \simeq \Pi_{n}\left(\operatorname{perf} A_{Q}\right),
$$

which holds if $n \geqslant 2$ and $\check{w}$ defines a simple singularity. As discussed in Section 3, the proof of $(1.5)$ in [EL17b] depends on the computation of the Hochschild cohomology of $\Pi_{n}\left(\operatorname{perf} A_{Q}\right)$, which was missing for type E cases in [EL17b] and is done in Section 5.

For $n=1$ not covered by Theorem 1.2, a quasi-equivalence of the full subcategory $\mathcal{F}\left(\check{w}^{-1}(0)\right)$ of $\mathcal{W}\left(\check{w}^{-1}(0)\right)$ consisting of (direct summands of bounded complexes of) compact Lagrangians and a category $\operatorname{perf} Z_{\mathbf{w}}$ equivalent to the full subcategory of $\operatorname{mf}\left(\mathbb{A}^{3}, \Gamma_{\mathbf{w}}, \mathbf{w}+x_{0} x_{1} x_{2}\right)$ consisting of homologically finite objects (that is, those $X$ satisfying $\operatorname{dim} \bigoplus_{i \in \mathbb{Z}} \operatorname{Ext}^{i}(X, Y)<\infty$ for any object $Y$ ) is given in [Hab20, Theorem 1.1].

As an application of (1.3), we compute the Hochschild cohomology group of the $n$-Calabi-Yau completion $\Pi_{n}\left(A_{Q}\right)$, also known as the derived n-preprojective algebra, of the path algebra $A_{Q}$ of any Dynkin quiver $Q$ for any $n \geqslant 1$. It is possible to compute the Hochschild homology along the same lines.

The zeroth cohomology of the derived 2-preprojective algebra is the preprojective algebra. The Hochschild homology and cohomology of the preprojective algebra of the path algebra of a Dynkin quiver is calculated in [ES98a, ES98b, ES98c, EE07]. Even the calculus structure in the sense of [GDT90, TT00] (which includes the Batalin-Vilkovisky structure and is known to be derived invariant [AK19]) is calculated in [Eu10], and it is an interesting problem to do the same for the derived $n$-preprojective algebra. Note that the preprojective algebra and the derived 2-preprojective algebra of a Dynkin quiver are very different. The derived 2-preprojective algebra of a Dynkin quiver is a smooth dg algebra, which has cohomology in every negative cohomological degree and moreover is not formal. In contrast, the preprojective algebra is always concentrated in cohomological degree 0 by definition, and the global dimension is infinite for a Dynkin quiver.

It follows from [Gan12, Theorem 1.1] combined with [CDGG17, Theorem 1.4], which builds on [Gan12, Gao17], that the closed-open map of any Weinstein manifold from the symplectic cohomology to the Hochschild cohomology of the wrapped Fukaya category is an isomorphism:

$$
\mathrm{SH}^{*}(M) \stackrel{\sim}{\rightarrow} \mathrm{HH}^{*}(\mathcal{W}(M)) .
$$

Hence, by Theorem 1.2, we see that the symplectic cohomology of the Milnor fiber $\check{w}^{-1}(1)$ of a simple singularity for $n \geqslant 2$ is isomorphic to $\mathrm{HH}^{*}\left(\Pi_{n}\left(A_{Q}\right)\right)$. This enables us to give an explicit computation of the symplectic cohomology of Milnor fibers of all simple singularities in a uniform way. Previous partial results computing symplectic cohomology for Milnor fibers of simple singularities appeared in [EL17b] for $A_{\ell}$ and $D_{\ell}$ in complex dimension 2 and in [KvK16, Ueb16] 


\section{SiMPLE SINGULARITIES}

for various versions of symplectic cohomology for certain higher-dimensional $A_{\ell}$-Milnor fibers for which an associated Morse-Bott spectral sequence yields computations. Our computation also shows that $\mathrm{HH}^{*}\left(\Pi_{1}\left(A_{Q}\right)\right)$ is not isomorphic to $\mathrm{SH}^{*}\left(\check{w}^{-1}(0)\right)$ given in [Hab20, Section 3.3], which is consistent with the failure of (1.5) for $n=1$.

This paper is organized as follows: In Section 2, we collect basic definitions and results on Calabi-Yau completions and trivial extension algebras. In Section 3, we recall the description of the wrapped Fukaya category of the Milnor fiber of a simple singularity for $n \geqslant 2$ in terms of the $n$-Calabi-Yau completion of a Dynkin quiver of the corresponding type. In Section 4, we prove (1.2) and (1.3). The computation of Hochschild cohomologies of the derived preprojective algebras of Dynkin quivers are given in Section 5 .

\section{Calabi-Yau completions and trivial extension algebras}

The $n$-Calabi-Yau completion (or the derived n-preprojective algebra) of a dg category $\mathscr{A}$ is defined in [Kel11, Section 4.1] as the tensor algebra

$$
\Pi_{n}(\mathscr{A}):=T_{\mathscr{A}}(\theta):=\mathscr{A} \oplus \theta \oplus \theta \otimes_{\mathscr{A}} \theta \oplus \cdots,
$$

where the $\mathscr{A}$-bimodule $\theta:=\Theta[n-1]$ is a shift of the inverse dualizing complex

$$
\Theta:=\operatorname{hom}_{\mathscr{A}} \mathrm{e}\left(\mathscr{A}, \mathscr{A}^{\mathrm{e}}\right) \text {. }
$$

A dg algebra is regarded as a dg category with one object. The Morita invariance of the Calabi-Yau completion shown in [Kel11, Proposition 4.2] implies that Calabi-Yau completion commutes with the operation of taking the dg category of perfect dg modules:

$$
\Pi_{n}(\operatorname{perf} \mathscr{A}) \simeq \operatorname{perf}\left(\Pi_{n} \mathscr{A}\right) .
$$

The Ginzburg dg algebra $\mathscr{G}_{Q}^{n}$ of a quiver $Q$ (without potential) is a model of the $n$-Calabi-Yau completion $\Pi_{n}\left(A_{Q}\right)$ of the path algebra $A_{Q}$, defined in [Kel11, Section 6.2] after [Gin06] as the path algebra of the graded quiver $\bar{Q}$ with same vertices as $Q$ and arrows consisting of

- the original arrows $g \in Q_{1}$ in degree 1 ,

- the opposite arrows $g^{*}$ for each arrow $g \in Q_{1}$ in degree $1-n$, and

- loops $h_{v}$ at each vertex $v \in Q_{0}$ in degree $1-n$,

equipped with the differential $d$ given by

$$
d g=d g^{*}=0 \quad \text { and } \quad d h=\sum_{g \in Q_{1}} g^{*} g-g g^{*},
$$

where $h=\sum_{v \in Q_{0}} h_{v}$.

The degree $n$ trivial extension algebra of a finite-dimensional algebra $A$ is defined as $A \oplus$ $A^{\vee}[-n]$ equipped with the multiplication $(a, f) \cdot(b, g)=(a b, a g+f b)$, where $A^{\vee}$ is the dual of $A$ as a vector space.

The degree $n$ trivial extension algebra $B_{Q}^{n}$ of the path algebra $A_{Q}$ of a Dynkin quiver $Q$ is the (derived) Koszul dual of $\mathscr{G}_{Q}^{n}$ in the sense that

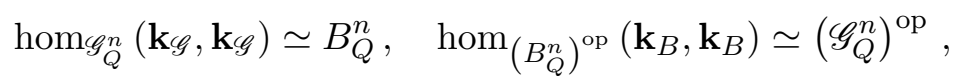

where $\mathbf{k}_{\mathscr{G}}:=\bigoplus_{v \in Q_{0}} S_{v}$ is the direct sum of simple left $\mathscr{G}_{Q}^{n}$-modules $S_{v}$ associated with vertices $v \in Q_{0}$, and similarly for $\mathbf{k}_{B}$ (see, for example, [EL17b, Theorem 23, Corollary 25]). 


\section{Y. LEKILI AND K. UEDA}

This Koszul duality implies an isomorphism

$$
\mathrm{HH}^{*}\left(\mathscr{G}_{Q}^{n}\right) \cong \mathrm{HH}^{*}\left(B_{Q}^{n}\right)
$$

of Hochschild cohomologies (see, for example, [FMT05, Theorem 1] and [Her19, Theorem 3.4]).

\section{The wrapped Fukaya category of the Milnor fiber of a simple singularity}

Let $\check{w}$ be one of the defining polynomials of a simple singularity and $M^{n}=\check{w}^{-1}(1)$ be the Milnor fiber, which we view as a Weinstein manifold where the Weinstein structure is induced by restriction from the ambient $\mathbb{C}^{n+1}$. It is well known that this Weinstein manifold is symplectomorphic (in fact, Weinstein homotopic) to the plumbing $X_{Q}$ of cotangent bundles of spheres $T^{*} S^{n}$ according to the Dynkin diagram $Q$ corresponding to the simple singularity. One way to see this is to verify it directly for $n=1$ and then use the fact that in higher dimensions, the Milnor fiber is obtained by stabilization-increasing the dimension corresponds to the suspension of the Lefschetz fibration [Sei10]. See also [Abo11] for an explicit construction of a symplectic structure on plumbings. This stabilization point of view also enables one to describe $M$ via Legendrian surgery. Namely, $M$ is obtained by attaching critical handles to a Legendrian link $\Lambda_{Q}^{n-1}$ on $\partial \mathbb{D}^{n}$ whose components are unknotted Legendrian spheres $S^{n-1}$ which are clasped together (as in Hopf link) according to the Dynkin diagram $Q$. The direct sum of co-cores to the critical handles (that is, cotangent fibers away from the plumbing region) form a generating object of the wrapped Fukaya category by the main theorem in [CDGG17], and the surgery formula of [BEE12, Ekh19] allows one to explicitly compute the endomorphism algebra of this generator as the Chekanov-Eliashberg algebra $\mathrm{CE}^{*}\left(\Lambda_{Q}^{n-1}\right)$.

This Chekanov-Eliashberg algebra was computed directly in the case $n=2$ in the paper [EL17b], and the resulting dg algebra was shown to be quasi-isomorphic to the derived multiplicative preprojective algebra of the corresponding Dynkin type. Moreover, working over $\mathbb{C}$, for $Q=A_{\ell}$ or $D_{\ell}$, it was shown in [EL17b, Theorem 13] that the derived multiplicative preprojective algebra of Dynkin type $Q$ is quasi-isomorphic to the Ginzburg algebra $\mathscr{G}_{Q}^{2}$, also known as the derived (additive) preprojective algebra of Dynkin type $Q$. It was conjectured in op. cit. that the same result holds for $Q=E_{6}, E_{7}, E_{8}$, and this is indeed so. The key ingredient for the proof of [EL17b, Theorem 13] to go through that was missing in the case $Q=E_{6}, E_{7}, E_{8}$ was the computation that

$$
\mathrm{HH}^{2}\left(\mathscr{G}_{Q}^{2}\right)^{s}=0 \text { for } s<0
$$

but this follows from computations given in Section 5 below.

For $n \geqslant 3$, one can do a direct computation in an analogous way, but we can also deduce this by the Koszul duality result given in [EL17a, Theorem 58], which shows that $\mathrm{CE}^{*}\left(\Lambda_{Q}^{n-1}\right)$ is the (derived) Koszul dual of the endomorphism algebra of the union of the core spheres of the plumbing. Notice that for $n \geqslant 3$, the polynomial $\check{w}$ is suspended at least twice; thus, the formality of the endomorphism algebra of vanishing cycles in the compact Fukaya category of $\check{w}^{-1}(1)$ follows automatically by [Sei10, Proposition 4.4] (the formality of the $A_{\infty}$-algebra $\mathcal{A}$ and an $\mathcal{A}$-bimodule $\mathcal{B} / \mathcal{A}$ in Seidel's notation is obvious in the case at hand, since $\Gamma$ is a tree and one can shift the objects to put all morphisms in degree 0$)$. Putting it all together, we conclude that $\mathrm{CE}^{*}\left(\Lambda_{Q}^{n-1}\right)$ is Koszul dual to the degree $n$ trivial extension algebra $B_{Q}^{n}$ of the path algebra $A_{Q}$ of a Dynkin quiver of the corresponding type (see also [Li19] for another example). 


\section{SiMPLE SINGULARITIES}

As a result of these computations, for $n \geqslant 2$ we have a quasi-isomorphism

$$
\mathrm{CE}^{*}\left(\Lambda_{Q}^{n-1}\right) \simeq \mathscr{G}_{Q}^{n}
$$

over $\mathbb{C}$, which implies a quasi-equivalence

$$
\mathcal{W}\left(\check{w}^{-1}(1)\right) \simeq \operatorname{perf\Pi } \Pi_{n}\left(A_{Q}\right)
$$

between the wrapped Fukaya category of $\breve{w}^{-1}(1)$ and the dg category of perfect modules over $\Pi_{n}\left(A_{Q}\right)$.

Remark 3.1. Note from [Sei01, Proposition 3.4] that $A_{Q}$ is derived equivalent to the FukayaSeidel category $\mathcal{F}(\check{w})$ of the LG-model $\check{w}: \mathbb{C}^{n+1} \rightarrow \mathbb{C}$. Thus $(3.2)$ shows that $\mathcal{W}\left(\check{w}^{-1}(1)\right)$ is the Calabi-Yau completion of $\mathcal{F}(\check{w})$ for $n \geqslant 2$. Although this relationship between $\mathcal{F}(\breve{w})$ and $\mathcal{W}\left(\check{w}^{-1}(1)\right)$ is not true in general, we expect it to hold when $\check{w}$ is a double suspension of an invertible polynomial whose Milnor fiber is a log Fano manifold, since one has

$$
\begin{aligned}
& \mathbf{w}\left(x_{1}, \ldots, x_{n-1}\right)+x_{n}^{2}+x_{n+1}^{2}+x_{0} \cdots x_{n+1} \\
& \quad=\mathbf{w}\left(x_{1}, \ldots, x_{n-1}\right)+\left(\sqrt{1-\frac{1}{4}\left(x_{0} \cdots x_{n-1}\right)^{2}} x_{n}\right)^{2}+\left(x_{n+1}+\frac{1}{2} x_{0} \cdots x_{n}\right)^{2}
\end{aligned}
$$

in $\mathbf{k} \llbracket x_{0}, \ldots, x_{n+1} \rrbracket$.

Remark 3.2. The isomorphism (3.1) remains true for $n \geqslant 3$ over an arbitrary commutative ring, but for $n=2$ we have to require that 2 be invertible for type $D_{\ell}, E_{6}, E_{7}, E_{8}$, that 3 be invertible for type $E_{6}, E_{7}, E_{8}$, and that 5 be invertible for type $E_{8}$. Otherwise, $\operatorname{CE}^{*}\left(\Lambda_{Q}\right)$ is quasi-isomorphic to the derived multiplicative preprojective algebra (see [EL19]), which is not quasi-isomorphic to the derived (additive) preprojective algebra $\Pi_{n}\left(A_{Q}\right)$.

\section{Matrix factorizations and Calabi-Yau completions}

Let $\Gamma$ be a subgroup of $\left(\mathbb{G}_{\mathrm{m}}\right)^{n+1}$ acting diagonally on $\mathbb{A}^{n+1}:=\operatorname{Spec} \mathbb{C}\left[x_{1}, \ldots, x_{n+1}\right]$. Assume that $\Gamma$ is a finite extension of the multiplicative group $\mathbb{G}_{\mathrm{m}}$, so that the group $\operatorname{Char}(\Gamma):=$ $\operatorname{Hom}\left(\Gamma, \mathbb{G}_{\mathrm{m}}\right)$ of characters of $\Gamma$ is an extension of a finite group by $\mathbb{Z}$. The coordinate ring $\mathbb{C}\left[x_{1}, \ldots, x_{n+1}\right]$ has a Char $(\Gamma)$-grading coming from the $\Gamma$-action on $\mathbb{A}^{n+1}$, and we set $\chi_{i}:=\operatorname{deg} x_{i}$ for $i \in\{1, \ldots, n+1\}$. Let $\mathbf{w} \in \mathbb{C}\left[x_{1}, \ldots, x_{n+1}\right]_{\chi}$ be a homogeneous element of degree $\chi \in \operatorname{Char}(\Gamma)$. Assume that $\mathbf{w}$ has an isolated critical point at the origin, so that the structure sheaf $\mathcal{O}_{0}$ of the origin split-generates mf $\left(\mathbb{A}^{n+1}, \mathbf{w}\right)$ by [KMVdB11, Proposition A.2] (see also [Orl11, Dyc11]). Let $R \subset \operatorname{Char}(\Gamma)$ be a set of representatives of the group $\operatorname{Char}(\Gamma) /(\chi)$, which we assume to be finite. Then $\mathcal{E}:=\bigoplus_{\rho \in R} \mathcal{O}_{0}(\rho)$ split-generates $\operatorname{mf}\left(\mathbb{A}^{n+1}, \Gamma, \mathbf{w}\right)$ since the autoequivalence $M \mapsto M(\chi)$ of $\operatorname{mf}\left(\mathbb{A}^{n+1}, \Gamma, \mathbf{w}\right)$ shifting the $\Gamma$-weight by $\chi$ is isomorphic to the functor $M \mapsto M[2]$ shifting the cohomological grading by 2 .

The $n$-Calabi-Yau completion of the dg Yoneda algebra $\mathscr{A}:=\operatorname{hom}(\mathcal{E}, \mathcal{E})$ is given by

$$
\Pi_{n}(\mathscr{A}):=\mathscr{A} \oplus \theta \oplus \theta \otimes \mathscr{A} \theta \oplus \cdots \simeq \bigoplus_{i=0}^{\infty} \operatorname{hom}\left(\mathcal{E}, \theta^{i}(\mathcal{E})\right),
$$

where $\theta=\Theta[n-1]$ as in Section 2, and we abuse notation and use the same symbol for an autoequivalence and its graph bimodule. Since $\Theta$ is the graph of the inverse Serre functor $\mathbb{S}^{-1}$, we have

$$
\theta=\mathbb{S}^{-1}[n-1] .
$$




\section{Y. LEKILI AND K. UEDA}

Now, as in [LU18, Section 2], we introduce another variable $x_{0}$ of degree $\chi_{0}:=\chi-\left(\chi_{1}+\cdots+\right.$ $\left.\chi_{n+1}\right)$ and consider the polynomial ring $\mathbb{C}\left[x_{0}, x_{1}, \ldots, x_{n+1}\right]$ in $n+2$ variables, which naturally contains $\mathbb{C}\left[x_{1}, \ldots, x_{n+1}\right]$ as a subring. One has

$$
\operatorname{mf}\left(\mathbb{A}^{n+2}, \mathbf{w}\right) \simeq \operatorname{mf}\left(\mathbb{A}^{1}, 0\right) \otimes \operatorname{mf}\left(\mathbb{A}^{n+1}, \mathbf{w}\right),
$$

for example, by the ungraded $(G=H=1)$ version of [BFK14, Lemma 3.52] with $v=0$; note that $\mathrm{mf}\left(\mathbb{A}^{1}, 0\right)$ is obtained from coh $\mathbb{A}^{1}$ by collapsing the cohomological grading to $\mathbb{Z} / 2 \mathbb{Z}$, and the tensor product of split-generators of $\operatorname{mf}\left(\mathbb{A}^{1}, 0\right)$ and $m f\left(\mathbb{A}^{n+1}, \mathbf{w}\right)$ gives a split-generator of $\operatorname{mf}\left(\mathbb{A}^{n+2}, \mathbf{w}\right)$ since the critical locus of $\mathbf{w}$ as a function on $\mathbb{A}^{n+2}$ is the product of $\mathbb{A}^{1}$ times that as a function on $\mathbb{A}^{n+1}$.

As shown in [IT13, Theorem 2.5], whose proof carries over directly to $\Gamma$-graded cases, graded Auslander-Reiten duality [AR87] implies that

$$
\mathbb{S}:=\left(\chi_{0}\right)[n-1]
$$

is a Serre functor on $\operatorname{mf}\left(\mathbb{A}^{n+1}, \Gamma, \mathbf{w}\right)$. It follows from (4.1) and (4.2) that

$$
\theta \simeq\left(-\chi_{0}\right)
$$

Let $\mathcal{F}$ be the generator of $\mathrm{mf}\left(\mathbb{A}^{n+2}, \Gamma, \mathbf{w}\right)$ obtained from the tensor product of the generator $\mathcal{E}$ of $\operatorname{mf}\left(\mathbb{A}^{n+1}, \Gamma, \mathbf{w}\right)$ and the generator $\mathbb{C}\left[x_{0}\right]$ of $\operatorname{coh} \mathbb{A}^{1}$. If we write both of the forgetful functors $\operatorname{mf}\left(\mathbb{A}^{n+1}, \Gamma, \mathbf{w}\right) \rightarrow \operatorname{mf}\left(\mathbb{A}^{n+1}, \mathbf{w}\right)$ and $\operatorname{mf}\left(\mathbb{A}^{n+2}, \Gamma, \mathbf{w}\right) \rightarrow \operatorname{mf}\left(\mathbb{A}^{n+2}, \mathbf{w}\right)$ as $\overline{(\bullet)}$, then one has

$$
\operatorname{hom}(\overline{\mathcal{F}}, \overline{\mathcal{F}}) \simeq \operatorname{hom}(\overline{\mathcal{E}}, \overline{\mathcal{E}}) \otimes \mathbb{C}\left[x_{0}\right] \simeq \bigoplus_{\rho \in \operatorname{Char}(\Gamma)} \operatorname{hom}(\mathcal{E}, \mathcal{E}(\rho)) \otimes \mathbb{C}\left[x_{0}\right]
$$

Since $\operatorname{deg}\left(x_{0}\right)=\chi_{0}$, by taking the $\Gamma$-invariant part of (4.4) and using (4.3), one obtains

$$
\operatorname{hom}(\mathcal{F}, \mathcal{F}) \simeq \bigoplus_{i=0}^{\infty} \operatorname{hom}\left(\mathcal{E}, \mathcal{E}\left(-i \chi_{0}\right)\right) \simeq \bigoplus_{i=0}^{\infty} \operatorname{hom}\left(\mathcal{E}, \theta^{i}(\mathcal{E})\right) \simeq \Pi_{n}(\mathscr{A})
$$

which shows the quasi-equivalence (1.3).

If $n$ is greater than 1 , then the degree of $x_{1} \cdots x_{n+1}$ in $\operatorname{Char}\left(\Gamma_{\mathbf{w}}\right) \otimes \mathbb{Q} \cong \mathbb{Q}$ is greater than the degree of $\mathbf{w}$, which is turn is greater than the degree of any element of the Jacobi ring

$$
\mathrm{Jac}_{\mathbf{w}}:=\mathbb{C}\left[x_{1}, \ldots, x_{n+1}\right] /\left(\partial_{x_{1}} \mathbf{w}, \ldots, \partial_{x_{n+1}} \mathbf{w}\right)
$$

of $\mathbf{w}$, and the proof of [AGV85, Section 12.6, Theorem] shows that the polynomial $\mathbf{w}+x_{0} \cdots x_{n+1}$ considered as an element of $\mathbb{C} \llbracket x_{0} \rrbracket \llbracket x_{1}, \ldots, x_{n+1} \rrbracket$ (that is, a formal one-parameter deformation of a formal germ of $\mathbf{w}$ ) is right equivalent to $\mathbf{w}$ by a formal coordinate change (that is, there exists a $\varphi \in$ Aut $_{\mathbb{C} \llbracket x_{0} \rrbracket} \mathbb{C} \llbracket x_{0} \rrbracket \llbracket x_{1}, \ldots, x_{n+1} \rrbracket$ such that $\left.\varphi^{*}\left(\mathbf{w}+x_{0} \cdots x_{n+1}\right)=\mathbf{w}\right)$. The proof moreover shows that one can choose $\varphi$ to be $\Gamma_{\mathbf{w}}$-equivariant, which implies that for any $i \in\{1, \ldots, n+1\}$, the coefficient $a_{i, m_{1}, \ldots, m_{n+1}}\left(x_{0}\right)$ of the expansion $\varphi^{*}\left(x_{i}\right)=\sum_{m_{1}, \ldots, m_{n+1}=0}^{\infty} a_{i, m_{1}, \ldots, m_{n+1}}\left(x_{0}\right) x_{1}^{m_{1}} \cdots x_{n+1}^{m_{n+1}}$ is a monomial in $x_{0}$ since the degree of $x_{0}$ in $\operatorname{Char}\left(\Gamma_{\mathbf{w}}\right) \otimes \mathbb{Q}$ is negative. In particular, one has $\varphi \in$ Aut $_{\mathbb{C}\left[x_{0}\right]} \mathbb{C}\left[x_{0}\right] \llbracket x_{1}, \ldots, x_{n+1} \rrbracket$. This means that the formal completion of $\left(\mathbb{A}^{n+2}, \mathbf{w}+x_{0} \cdots x_{n+1}\right)$ along $x_{1}=\cdots=x_{n+1}=0$ is isomorphic to that of $\left(\mathbb{A}^{n+2}, \mathbf{w}\right)$ as a pair of a formal scheme and a regular function on it, so that

$$
\operatorname{mf}\left(\mathbb{A}^{n+2}, \Gamma_{\mathbf{w}}, \mathbf{w}+x_{0} \cdots x_{n+1}\right) \simeq \operatorname{mf}\left(\mathbb{A}^{n+2}, \Gamma_{\mathbf{w}}, \mathbf{w}\right)
$$

by [Orl11, Theorem 2.10], and the quasi-equivalence (1.2) is proved. 


\section{SiMPLE SINGULARITIES}

\section{Hochschild cohomology of the derived $n$-preprojective algebra}

\subsection{Hochschild cohomology via matrix factorizations}

We use the same notation as in Section 4 and set

$$
V:=\mathbb{C} x_{0} \oplus \mathbb{C} x_{1} \oplus \cdots \oplus \mathbb{C} x_{n+1} .
$$

For $\gamma \in \Gamma$, let $V_{\gamma}$ be the subspace of $\gamma$-invariant elements in $V$, let $S_{\gamma}$ be the symmetric algebra of $V_{\gamma}$, let $\mathbf{w}_{\gamma}$ be the restriction of $\mathbf{w}$ to Spec $S_{\gamma}$, and let $N_{\gamma}$ be the $\Gamma$-stable complement of $V_{\gamma}$ in $V$ so that $V \cong V_{\gamma} \oplus N_{\gamma}$ as a $\Gamma$-module. Then [Dyc11, CT13, Seg13, BFK14] (cf. also [LU18, Theorem 3.1]) shows that $\mathrm{HH}^{t}\left(\operatorname{mf}\left(\mathbb{A}^{n+2}, \Gamma, \mathbf{w}\right)\right)$ is isomorphic to

$$
\bigoplus_{\substack{\gamma \in \operatorname{ker} \chi, l \geqslant 0 \\ t-\operatorname{dim} N_{\gamma}=2 u}}\left(H^{-2 l}\left(d \mathbf{w}_{\gamma}\right) \otimes \Lambda^{\operatorname{dim} N_{\gamma}} N_{\gamma}^{\vee}\right)_{(u+\ell) \chi} \oplus \bigoplus_{\substack{\gamma \in \operatorname{ker} \chi, l \geqslant 0 \\ t-\operatorname{dim} N_{\gamma}=2 u+1}}\left(H^{-2 l-1}\left(d \mathbf{w}_{\gamma}\right) \otimes \Lambda^{\operatorname{dim} N_{\gamma}} N_{\gamma}^{\vee}\right)_{(u+\ell+1) \chi} .
$$

Here $H^{i}\left(d \mathbf{w}_{\gamma}\right)$ is the $i$ th cohomology of the Koszul complex

$$
C^{*}\left(d \mathbf{w}_{\gamma}\right):=\left\{\cdots \rightarrow \Lambda^{2} V_{\gamma}^{\vee} \otimes S_{\gamma}(-2 \chi) \rightarrow V_{\gamma}^{\vee} \otimes S_{\gamma}(-\chi) \rightarrow S_{\gamma}\right\},
$$

where the rightmost term $S_{\gamma}$ sits in cohomological degree 0 and the differential is the contraction with $d \mathbf{w}_{\gamma} \in\left(V_{\gamma} \otimes S_{\gamma}\right)_{\chi}$.

If $\mathbf{w}_{\gamma}$ has an isolated critical point at the origin, then the cohomology of (5.2) is concentrated in degree 0 , so that only the summand

$$
\left(\mathrm{Jac}_{\mathbf{w}_{\gamma}} \otimes \Lambda^{\operatorname{dim} N_{\gamma}} N_{\gamma}^{\vee}\right)_{u \chi}
$$

with $l=0$ in (5.1) contributes to $\mathrm{HH}^{2 u+\operatorname{dim} N_{\gamma}}$.

If $V_{\gamma}$ contains $\mathbb{C} x_{0}$, then the Koszul complex $C^{*}\left(d \mathbf{w}_{\gamma}\right)$ is isomorphic to the tensor product of $C^{*}\left(d \mathbf{w}_{\gamma}^{\prime}\right)$ and the complex $\left\{\mathbb{C} x_{0}^{\vee} \otimes \chi^{\vee} \otimes \mathbb{C}\left[x_{0}\right] \rightarrow \mathbb{C}\left[x_{0}\right]\right\}$ concentrated in cohomological degree $[-1,0]$ with the zero differential, where $\mathbf{w}_{\gamma}^{\prime}$ is the restriction of $\mathbf{w}$ to the complement $V_{\gamma}^{\prime}$ of $\mathbb{C} x_{0}$ in $V_{\gamma}$. If $\mathbf{w}_{\gamma}^{\prime}$ has an isolated critical point at the origin, then $C^{*}\left(d \mathbf{w}_{\gamma}^{\prime}\right)$ is quasi-isomorphic to $\operatorname{Jac}_{\mathbf{w}_{\gamma}^{\prime}}$ concentrated in cohomological degree 0 , so that only the summands

$$
\left(\mathrm{Jac}_{\mathbf{w}_{\gamma}^{\prime}} \otimes \mathbb{C}\left[x_{0}\right] \otimes \Lambda^{\operatorname{dim} N_{\gamma}} N_{\gamma}^{\vee}\right)_{u \chi}
$$

and

$$
\left(\mathbb{C} x_{0}^{\vee} \otimes \mathrm{Jac}_{\mathbf{w}_{\gamma}^{\prime}} \otimes \mathbb{C}\left[x_{0}\right] \otimes \Lambda^{\operatorname{dim} N_{\gamma}} N_{\gamma}^{\vee}\right)_{u \chi}
$$

with $l=0$ in (5.1) contribute to $\mathrm{HH}^{2 u+\operatorname{dim} N_{\gamma}}$ and $\mathrm{HH}^{2 u+\operatorname{dim} N_{\gamma}+1}$, respectively.

Remark 5.1. Although (5.1) may not look identical to [BFK14, Theorem 1.2], the proof in [BFK14, Section 5] actually shows (5.1). One way to think about (5.1) is the following: If we set $\mathbf{w}=0$ and forget $\Gamma$, then the Hochschild-Kostant-Rosenberg theorem gives a quasi-isomorphism of the Hochschild cochain complex of $\operatorname{coh} \mathbb{A}^{n+2}$ and

$$
S \rightarrow V^{\vee} \otimes S \rightarrow \Lambda^{2} V^{\vee} \otimes S \rightarrow \cdots
$$

as complexes of $\mathbb{C}$-vector spaces (which lifts to a quasi-isomorphism of $L_{\infty}$-algebras by the Kontsevich formality). If we introduce the potential $\mathbf{w}$, then the complex (5.5) acquires an additional differential $\Lambda^{i} V^{\vee} \otimes S \rightarrow \Lambda^{i-1} V^{\vee} \otimes S$ defined as the contraction with $d \mathbf{w} \in V \otimes S$, which decreases the cohomological grading by 1 so that the cohomological grading is collapsed to $\mathbb{Z} / 2 \mathbb{Z}$. The introduction of $\Gamma$ lifts the grading to $\mathbb{Z}$ again and produces 'twisted sectors' from the orbifold HKR 


\section{Y. LEKILI AND K. UEDA}

theorem, leading to (5.1); recall the isomorphism $(\chi) \simeq[2]$ of endofunctors of $\mathrm{mf}\left(\mathbb{A}^{n+2}, \Gamma, \mathbf{w}\right)$ and the orbifold HKR theorem

$$
\mathrm{HH}^{*}([X / G])=\left(\bigoplus_{g \in G} \bigoplus_{p+q=*} H^{p-\operatorname{codim} X^{g}}\left(X^{g}, \Lambda^{q} T_{X^{g}} \otimes \Lambda^{\operatorname{codim} X^{g}} N_{X^{g} / X}\right)\right)_{G}
$$

for global quotients appearing, for example, in [ACH19, Corollary 1.17].

Remark 5.2. The Hochschild cohomology of a graded algebra $B$ (with no differential) has a bigrading such that

$$
\mathrm{HH}^{r+s}(B)^{s}:=\operatorname{Ext}_{B^{\mathrm{op}} \otimes B}^{r}(B, B[s]) .
$$

When $B$ is the trivial extension algebra $B^{n}$ of a finite-dimensional algebra $A$, by introducing a $\mathbb{G}_{\mathrm{m}}$-action on $B^{n}$ such that $A$ has weight 0 and $A^{\vee}[-n]$ has weight $n$, the $s$-grading on $\mathrm{HH}^{*}\left(B^{n}\right)$ can be described as the weight of the induced $\mathbb{G}_{\mathrm{m}}$-action.

For any positive integer $m$, the underlying ungraded algebra of the trivial extension algebras $B^{m n}$ is isomorphic to $B^{n}$, and only the cohomological gradings are different; that of the former is $m$ times that of the latter. It follows that one has an isomorphism

$$
\mathrm{HH}^{r+m s}\left(B^{m n}\right)^{m s} \cong \mathrm{HH}^{r+s}\left(B^{n}\right)^{s}
$$

of vector spaces for any positive integer $m$ such that the parities of $n$ and $m n$ are the same (note that the signs in the Hochschild complex depend on the parity of the cohomological grading).

When $Q$ is a Dynkin quiver, one can transport the $\mathbb{G}_{\mathrm{m}}$-action on $B_{Q}^{n}$ to $\mathscr{G}_{Q}^{n}$ through the Koszul duality (2.1), so that $g$ for $g \in Q_{1}$ has weight 0 , while $g^{*}$ for $g \in Q_{1}$ has weight $-n$, and $h_{v}$ for $v \in Q_{0}$ has weight $-n$. This makes the isomorphism (2.2) $\mathbb{G}_{\mathrm{m}}$-equivariant, so that the $\mathbb{G}_{\mathrm{m}}$-weights on both sides agree.

Since $\mathbf{w}$ does not depend on $x_{0}$, the $\mathbb{G}_{\mathrm{m}}$-action on $\mathbb{A}^{n+2}$ such that the weight of $x_{i}$ is $-n$ for $i=0$ and 0 for $i \in\{1, \ldots, n+1\}$ keeps $\mathbf{w}$ invariant. This induces a $\mathbb{G}_{\mathrm{m}}$-action on $\mathrm{mf}\left(\mathbb{A}^{n+2}, \Gamma, \mathbf{w}\right)$, and hence on $B_{Q}^{n}$, whose weight is 0 on $A_{Q}$ and $n$ on $A_{Q}^{\vee}[-n]$ just as in [LU18]. This allows us to compute the $s$-grading on $\mathrm{HH}^{*}\left(B_{Q}^{n}\right)$ as the $\mathbb{G}_{\mathrm{m}}$-weight on (5.1). This $\mathbb{G}_{\mathrm{m}}$-action is mirror to the one introduced in [SS12] and studied further for type A Milnor fibers in [Sei12].

\subsection{Type $A_{\ell}$}

Consider the case

$$
\begin{gathered}
\mathbf{w}=x_{1}^{\ell+1}+x_{2}^{2}+\cdots+x_{n+1}^{2} \in \mathbb{C}\left[x_{0}, x_{1}, \ldots, x_{n+1}\right], \\
\Gamma=\Gamma_{\mathbf{w}}:=\left\{\gamma=\left(t_{0}, t_{1}, \ldots, t_{n+1}\right) \in\left(\mathbb{G}_{\mathrm{m}}\right)^{n+2} \mid t_{1}^{\ell+1}=t_{2}^{2}=\cdots=t_{n+1}^{2}=t_{0} t_{1} \cdots t_{n+1}\right\},
\end{gathered}
$$

so that ker $\chi \cong \boldsymbol{\mu}_{\ell+1} \times\left(\boldsymbol{\mu}_{2}\right)^{n}$ and $\operatorname{Char}(\Gamma)$ is generated by $\chi$ and $\chi_{i}=\operatorname{deg} x_{i}$ for $i \in\{0, \ldots, n+1\}$ with relations

$$
\chi=(\ell+1) \chi_{1}=2 \chi_{2}=\cdots=2 \chi_{n+1}=\chi_{0}+\cdots+\chi_{n+1} .
$$

5.2.1 For any $\gamma \in \operatorname{ker} \chi$, one has

$$
\mathrm{Jac}_{\mathbf{w}_{\gamma}}= \begin{cases}\mathbb{C}\left[x_{0}\right] \otimes \mathbb{C}\left[x_{1}\right] /\left(x_{1}^{\ell}\right), & \mathbb{C} x_{0} \oplus \mathbb{C} x_{1} \subset V_{\gamma}, \\ \mathbb{C}\left[x_{0}\right], & \mathbb{C} x_{0} \subset V_{\gamma} \text { and } \mathbb{C} x_{1} \not \subset V_{\gamma}, \\ \mathbb{C}\left[x_{1}\right] /\left(x_{1}^{\ell}\right), & \mathbb{C} x_{0} \not \subset V_{\gamma} \text { and } \mathbb{C} x_{1} \subset V_{\gamma}, \\ \mathbb{C}, & \text { otherwise }\end{cases}
$$




\section{SiMPLE SINGULARITIES}

If we write an element of $\mathrm{Jac}_{\mathbf{w}_{\gamma}} \otimes \Lambda^{\operatorname{dim} N_{\gamma}} N_{\gamma}^{\vee}$ as $x_{0}^{k_{0}} x_{1}^{k_{1}} \otimes x_{j_{1}}^{\vee} \wedge x_{j_{2}}^{\vee} \wedge \cdots \wedge x_{j_{s}}^{\vee}$, where $k_{0}=0$ if $\mathbb{C} x_{0} \not \subset V_{\gamma}$ and $k_{1}=0$ if $\mathbb{C} x_{1} \not \subset V_{\gamma}$, then its degree is given by

$$
k_{0} \chi_{0}+k_{1} \chi_{1}-\chi_{j_{1}}-\cdots-\chi_{j_{s}},
$$

which can be proportional to $\chi$ only if $V_{\gamma}$ is either $V, \mathbb{C} x_{0} \oplus \mathbb{C} x_{1}, \mathbb{C} x_{0}$, or 0 . We now deal with each of these cases in turn.

5.2.2 One has $V_{\gamma}=V$ if and only if $\gamma$ is the identity element. The degree of $x_{0}^{k_{0}} x_{1}^{k_{1}} \in \mathrm{Jac}_{\mathbf{w}}$ is

$$
k_{0} \chi-\left(k_{0}-k_{1}\right) \chi_{1}-k_{0} \chi_{2}-\cdots-k_{0} \chi_{n+1},
$$

which is proportional to $\chi$ if and only if $k_{0}$ is even and $\ell+1$ divides $k_{0}-k_{1}$. Such an element can be written as $\boldsymbol{a}_{k, m}:=x_{0}^{k+m(\ell+1)} x_{1}^{k}$, where $k \in\{0, \ldots, \ell-1\}$ and $m \in \mathbb{N}$ satisfies

- if $\ell$ is even, then the parities of $k$ and $m$ agree, and

- if $\ell$ is odd, then $k$ is even.

Since

$$
\begin{aligned}
\operatorname{deg}\left(x_{0}^{k+m(\ell+1)} x_{1}^{k}\right) & =(k+m(\ell+1)) \chi-m \chi-\frac{1}{2}(k+m(\ell+1)) n \chi \\
& =\left((k+m \ell)-\frac{1}{2}(k+m(\ell+1)) n\right) \chi,
\end{aligned}
$$

the element $x_{0}^{k+m(\ell+1)} x_{1}^{k}$ for such $(k, m)$ contributes $\mathbb{C}((k+m(\ell+1)) n)$ to $\mathrm{HH}^{t}$ for $t=2(k+$ $m \ell)-(k+m(\ell+1)) n$ by (5.3). Similarly, for each such $(k, m)$, the element

$$
\boldsymbol{\alpha}_{k, m}:=x_{0}^{\vee} \otimes x_{0}^{k+m(\ell+1)+1} x_{1}^{k} \in \mathbb{C} x_{0}^{\vee} \otimes \mathrm{Jac}_{\mathbf{w}}
$$

contributes $\mathbb{C}((k+m(\ell+1)) n)$ to $\mathrm{HH}^{t+1}$ for $t=2(k+m \ell)-(k+m(\ell+1)) n$ by $(5.4)$.

5.2.3 One has $V_{\gamma}=\mathbb{C} x_{0} \oplus \mathbb{C} x_{1}$ if and only if $n$ is even and $\gamma=(1,1,-1, \ldots,-1)$. The degree of $x_{0}^{k_{0}} x_{1}^{k_{1}} \otimes x_{2}^{\vee} \wedge \cdots \wedge x_{n+1}^{\vee} \in \mathrm{Jac}_{\mathbf{w}_{\gamma}} \otimes \Lambda^{\operatorname{dim} N_{\gamma}} N_{\gamma}^{\vee}$ is given by

$$
k_{0} \chi+\left(k_{1}-k_{0}\right) \chi_{1}-\left(k_{0}+1\right) \chi_{2}-\cdots-\left(k_{0}+1\right) \chi_{n+1},
$$

which is proportional to $\chi$ if and only if $k_{0}$ is odd and $\ell+1$ divides $k_{1}-k_{0}$. Such an element can be written as

$$
\boldsymbol{a}_{k, m}:=x_{0}^{k+m(\ell+1)} x_{1}^{k} \otimes x_{2}^{\vee} \wedge \cdots \wedge x_{n+1}^{\vee},
$$

where $k \in\{0, \ldots, \ell-1\}$ and $m \in \mathbb{N}$ satisfies

- if $\ell$ is even, then the parities of $k$ and $m$ differ, and

- if $\ell$ is odd, then $k$ is odd.

Since the degree of this element is $\left((k+m \ell)-\frac{1}{2}(k+m(\ell+1)+1) n\right) \chi$, each such $(k, m)$ contributes $\mathbb{C}((k+m(\ell+1)) n)$ to $\mathrm{HH}^{t}$ for

$$
\begin{aligned}
t & =2\left((k+m \ell)-\frac{1}{2}(k+m(\ell+1)+1) n\right)+\operatorname{dim} N_{\gamma} \\
& =2(k+m \ell)-(k+m(\ell+1)) n .
\end{aligned}
$$

Similarly, for each such $(k, m)$, there is an element $\boldsymbol{\alpha}_{k, m}$ contributing $\mathbb{C}((k+m(\ell+1)) n)$ to $\mathrm{HH}^{t+1}$ for $t=2(k+m \ell)-(k+m(\ell+1)) n$. 


\section{Y. LEKILI AND K. UEDA}

5.2.4 One has $V_{\gamma}=\mathbb{C} x_{0}$ if and only if both $\ell$ and $n$ are odd and $\gamma=(1,-1, \ldots,-1)$. The degree of $x_{0}^{k_{0}} \otimes x_{1}^{\vee} \wedge \cdots \wedge x_{n+1}^{\vee} \in \mathrm{Jac}_{\mathbf{w}_{\gamma}} \otimes \Lambda^{\operatorname{dim} N_{\gamma}} N_{\gamma}^{\vee}$ is given by

$$
k_{0} \chi-\left(k_{0}+1\right) \chi_{1}-\left(k_{0}+1\right) \chi_{2}-\cdots-\left(k_{0}+1\right) \chi_{n+1},
$$

which is proportional to $\chi$ if and only if $\ell+1$ divides $k_{0}+1$. Such an element can be written as

$$
\boldsymbol{b}_{m}:=x_{0}^{m(\ell+1)-1} \otimes x_{1}^{\vee} \wedge \cdots \wedge x_{n+1}^{\vee}
$$

for $m \in \mathbb{N} \backslash\{0\}$. Since the degree of this element is $\left((m \ell-1)-\frac{1}{2} m(\ell+1) n\right) \chi$, each such element contributes $\mathbb{C}((m(\ell+1)-1) n)$ to $\mathrm{HH}^{t}$ for

$$
t=2\left((m \ell-1)-\frac{1}{2} m(\ell+1) n\right)+\operatorname{dim} N_{\gamma}=(2 m \ell-1)-(m(\ell+1)-1) n .
$$

Similarly, for each $m \in \mathbb{N}$, the element

$$
\boldsymbol{\beta}_{m}:=x_{0}^{\vee} \otimes x_{0}^{m(\ell+1)} \otimes x_{1}^{\vee} \wedge \cdots \wedge x_{n+1}^{\vee} \in \mathbb{C} x_{0}^{\vee} \otimes \operatorname{Jac}_{\mathbf{w}_{\gamma}} \otimes \Lambda^{\operatorname{dim} N_{\gamma}} N_{\gamma}^{\vee}
$$

contributes $\mathbb{C}(m(\ell+1)-1) n)$ to $\mathrm{HH}^{t+1}$ for $t=(2 m \ell-1)-(m(\ell+1)-1) n$.

5.2.5 For $\gamma=\left(t_{0}, \ldots, t_{n+1}\right) \in \operatorname{ker} \chi$, one has $V_{\gamma}=0$ if and only if $t_{i} \neq 1$ for all $i \in$ $\{0, \ldots, n+1\}$. This is the case if and only if $t_{2}=\cdots=t_{n+1}=-1, t_{1} \in \boldsymbol{\mu}_{\ell+1} \backslash\{1\}$, and

$$
t_{0}=(-1)^{n} t_{1}^{-1} \neq 1 \text {. }
$$

If $n$ is odd, then (5.6) holds if and only if $t_{1} \neq-1$, so that the number of such $\gamma$ is $\ell$ if $\ell$ is even and $\ell-1$ if $\ell$ is odd. If $n$ is even, then (5.6) always holds, and the number of such $\gamma$ is $\ell$. Each such $\gamma$ contributes $\mathbb{C}(-n)$ to $\mathrm{HH}^{n}$.

5.2.6 To sum up, the Hochschild cohomology group has a basis consisting of the following elements:

- $\boldsymbol{a}_{k, m}$ of degree $2(k+m \ell)-(k+m(\ell+1)) n$ and weight $-(k+m(\ell+1)) n$, and $\boldsymbol{\alpha}_{k, m}$ of degree $2(k+m \ell)-(k+m(\ell+1)) n+1$ and weight $-(k+m(\ell+1)) n$, where

$*$ if $n$ is even, then $(k, m)$ runs over $\{0, \ldots, \ell-1\} \times \mathbb{N}$, and

$*$ if $n$ is odd, then $(k, m)$ runs over those pairs in $\{0, \ldots, \ell-1\} \times \mathbb{N}$ for which

- the parities of $k$ and $m$ agree if $\ell$ is even,

. $k$ is even if $\ell$ is odd;

- if both $n$ and $\ell$ are odd,

* $\boldsymbol{b}_{m}$ of degree $2 m \ell-1-(m(\ell+1)-1) n$ and weight $-(m(\ell+1)-1) n$ for $m \in \mathbb{N} \backslash\{0\}$, and

* $\boldsymbol{\beta}_{m}$ of degree $2 m \ell-(m(\ell+1)-1) n$ and weight $-(m(\ell+1)-1) n$ for $m \in \mathbb{N}$; and

- $\boldsymbol{s}_{h}$ of degree $n$ and weight $n$, where $h$ runs over

$*\{1,2, \ldots, \ell-1\}$ if both $\ell$ and $n$ are odd,

$*\{1,2, \ldots, \ell\}$ otherwise.

5.2.7 As an example, consider the case $\ell=1$. Note that the $A_{1}$-Milnor fiber is symplectomorphic to the cotangent bundle $T^{*} S^{n}$. The Hochschild cohomology group in this case is spanned by

- $\boldsymbol{a}_{0, m}$ for $m \in \mathbb{N}$ of degree $-2 m(n-1)$ and weight $-2 m n$,

- $\boldsymbol{\alpha}_{0, m}$ for $m \in \mathbb{N}$ of degree $-2 m(n-1)+1$ and weight $-2 m n$, 


\section{SiMPLE SINGULARITIES}

and, if $n$ is odd, in addition to the above,

- $\boldsymbol{b}_{m}$ for $m \in \mathbb{N} \backslash\{0\}$ of degree $-(2 m-1)(n-1)$ and weight $-(2 m-1) n$,

$-\boldsymbol{\beta}_{m}$ for $m \in \mathbb{N}$ of degree $-(2 m-1)(n-1)+1$ and weight $-(2 m-1) n$,

and, if $n$ is even, in addition to the above,

$-s_{1}$ of degree $n$ and weight $n$.

This is consistent with the isomorphism

$$
\mathrm{SH}^{*}\left(T^{*} S^{n}\right) \cong H_{n-*}\left(\mathscr{L} S^{n}\right),
$$

which is a special case of the isomorphism between the symplectic cohomology of the cotangent bundle and the homology of the free loop space [Vit18, Theorem 3.1] (see, for example, [CJY04, Theorem 2] for the homology of the free loop space of spheres).

Another example is the case when $n=2$ and $\ell$ is arbitrary. In this case, $\operatorname{SH}^{*}\left(\check{w}^{-1}(1)\right)$ was computed in [EL17b] as a bigraded ring. This is compatible with the computation given here.

\subsection{Type $D_{\ell}$}

The Berglund-Hübsch transform of the invertible polynomial

$$
\check{\mathbf{v}}=y_{1}^{\ell-1}+y_{1} y_{2}^{2}+y_{3}^{2}+\cdots+y_{n+1}^{2}
$$

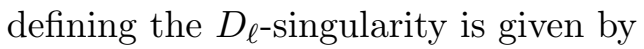

$$
\mathbf{v}=y_{1}^{\ell-1} y_{2}+y_{2}^{2}+\cdots+y_{n+1}^{2},
$$

and one has

$$
\Gamma_{\mathbf{v}}=\left\{\gamma=\left(t_{1}, \ldots, t_{n+1}\right) \in\left(\mathbb{G}_{\mathrm{m}}\right)^{n+1} \mid t_{1}^{\ell-1} t_{2}=t_{2}^{2}=\cdots=t_{n+1}^{2}\right\} .
$$

By completing the square and rescaling, one has $\mathbf{v}(y)=\mathbf{w}(x(y)) t$, where

$$
x_{1}=(-1 / 4)^{1 /(2 n-2)} y_{1}, \quad x_{2}=y_{2}+\frac{1}{2} y_{1}^{\ell-1}, \quad x_{3}=y_{3}, \quad \ldots, \quad x_{n+1}=y_{n+1},
$$

and $\mathbf{w}=x_{1}^{2 \ell-2}+x_{2}^{2}+\cdots+x_{n+1}^{2}$. Although the change of variables (5.7) is neither linear nor diagonal, the induced action of $\Gamma_{\mathbf{v}}$ on $\operatorname{Spec} \mathbb{C}\left[x_{1}, \ldots, x_{n+1}\right]$ remains linear and diagonal, so that one can identify $\Gamma_{\mathbf{v}}$ with a proper subgroup of $\Gamma_{\mathbf{w}}$.

Therefore, we will work with

$$
\mathbf{w}=x_{1}^{2 \ell-2}+x_{2}^{2}+\cdots+x_{n+1}^{2} \in \mathbb{C}\left[x_{0}, x_{1}, \ldots, x_{n+1}\right]
$$

with the non-maximal group

$$
\Gamma=\left\{\gamma=\left(t_{0}, t_{1}, \ldots, t_{n+1}\right) \in\left(\mathbb{G}_{\mathrm{m}}\right)^{n+2} \mid t_{1}^{\ell-1} t_{2}=t_{2}^{2}=\cdots=t_{n+1}^{2}=t_{0} t_{1} \cdots t_{n+1}\right\} .
$$

One has $\operatorname{ker} \chi \cong \boldsymbol{\mu}_{2 \ell-2} \times\left(\boldsymbol{\mu}_{2}\right)^{n-1}$, and $\operatorname{Char}(\Gamma)$ is generated by $\chi$ and $\chi_{i}=\operatorname{deg} x_{i}$ for $i \in$ $\{0, \ldots, n+1\}$ with relations

$$
\chi=(\ell-1) \chi_{1}+\chi_{2}=2 \chi_{2}=\cdots=2 \chi_{n+1}=\chi_{0}+\cdots+\chi_{n+1} .
$$

The relations (5.8) imply

$$
\begin{gathered}
\chi_{2}=\chi-(\ell-1) \chi_{1}, \quad \chi=(2 \ell-2) \chi_{1}, \\
\chi_{0}=\chi-\chi_{1}-\cdots-\chi_{n}=(\ell-2) \chi_{1}-\chi_{3}-\cdots-\chi_{n+1} .
\end{gathered}
$$




\section{Y. LEKILI AND K. UEDA}

5.3.1 For any $\gamma=\left(t_{0}, \ldots, t_{n+1}\right) \in \operatorname{ker} \chi$, one has

$$
\mathrm{Jac}_{\mathbf{w}_{\gamma}}= \begin{cases}\mathbb{C}\left[x_{0}\right] \otimes \mathbb{C}\left[x_{1}\right] /\left(x_{1}^{2 \ell-3}\right), & \mathbb{C} x_{0} \oplus \mathbb{C} x_{1} \subset V_{\gamma}, \\ \mathbb{C}\left[x_{0}\right], & \mathbb{C} x_{0} \subset V_{\gamma} \text { and } \mathbb{C} x_{1} \not \subset V_{\gamma}, \\ \mathbb{C}\left[x_{1}\right] /\left(x_{1}^{2 \ell-3}\right), & \mathbb{C} x_{0} \not \subset V_{\gamma} \text { and } \mathbb{C} x_{1} \subset V_{\gamma}, \\ \mathbb{C}, & \text { otherwise } .\end{cases}
$$

If we write an element of $\mathrm{Jac}_{\mathbf{w}_{\gamma}} \otimes \Lambda^{\operatorname{dim} N_{\gamma}} N_{\gamma}^{\vee}$ as $x_{0}^{k_{0}} x_{1}^{k_{1}} \otimes x_{j_{1}}^{\vee} \wedge x_{j_{2}}^{\vee} \wedge \cdots \wedge x_{j_{s}}^{\vee}$, where $k_{0}=0$ if $\mathbb{C} x_{0} \not \subset V_{\gamma}$ and $k_{1}=0$ if $\mathbb{C} x_{1} \not \subset V_{\gamma}$, then its degree is given by

$$
k_{0} \chi_{0}+k_{1} \chi_{1}-\chi_{j_{1}}-\cdots-\chi_{j_{s}}
$$

which can be proportional to $\chi$ only if $V_{\gamma} \cap\left(\mathbb{C} x_{3} \oplus \cdots \oplus \mathbb{C} x_{n+1}\right)$ is either $\mathbb{C} x_{3} \oplus \cdots \oplus \mathbb{C} x_{n+1}$ or 0 , that is,

$$
t_{3}=\cdots=t_{n+1}= \pm 1
$$

We will assume this condition for the rest of Section 5.3.

5.3.2 One has $\gamma=\left(t_{0}, \ldots, t_{n+1}\right) \in$ ker $\chi$ if and only if

$$
t_{1}^{\ell-1} t_{2}=t_{2}^{2}=\cdots=t_{n+1}^{2}=t_{0} t_{1} \cdots t_{n+1}=1 .
$$

If $t_{0}=1$, then one has $t_{2}^{2}=\cdots=t_{n+1}^{2}=t_{1} \cdots t_{n+1}=1$, so that $t_{1}=\left(t_{2} \cdots t_{n+1}\right)^{-1}= \pm 1$.

If $t_{1}=1$, then one has $t_{2}=t_{2}^{2}$, so that $t_{2}=1$ and $t_{3}^{2}=\cdots=t_{n+1}^{2}=t_{0} t_{3} \cdots t_{n+1}=1$. Under the assumption (5.9), one has $t_{0}=1$ if and only if $\left(t_{3}\right)^{n-1}=1$, that is, $t_{3}=1$ or $n$ is even.

If $t_{1}=-1$, then $t_{2}=(-1)^{\ell-1}$, and one has $t_{0}=1$ if and only if $(-1)^{\ell} t_{3}^{n-1}=1$. It follows that

- $V_{\gamma}$ contains $\mathbb{C} x_{0}$ if and only if

* $\gamma=(1, \ldots, 1)$, where $V_{\gamma}=V$;

$* \gamma=(1,1,1,-1, \ldots,-1)$ with odd $n$, where $V_{\gamma}=\mathbb{C} x_{0} \oplus \mathbb{C} x_{1} \oplus \mathbb{C} x_{2} ;$

$* \gamma=(1,-1,-1,1, \ldots, 1)$ with even $\ell$, where $V_{\gamma}=\mathbb{C} x_{0} \oplus \mathbb{C} x_{3} \oplus \cdots \oplus \mathbb{C} x_{n+1}$;

$* \gamma=(1,-1,-1,-1, \ldots,-1)$ with even $\ell$ and odd $n$, where $V_{\gamma}=\mathbb{C} x_{0}$; or

$* \gamma=(1,-1,1,-1, \ldots,-1)$ with odd $\ell$ and even $n$, where $V_{\gamma}=\mathbb{C} x_{0} \oplus \mathbb{C} x_{2}$.

5.3.3 Note for later use that the smallest positive integer $k$ such that the degree of $x_{0}^{k}$ is proportional to $\chi$ is $2 \ell-2$. One has

$$
\operatorname{deg} x_{0}^{2 \ell-2}=(2 \ell-2)\left(\chi-\chi_{1}-\cdots-\chi_{n+1}\right)=((2 \ell-3)-(\ell-1) n) \chi .
$$

5.3.4 One has $V_{\gamma}=V$ if and only if $\gamma$ is the identity element. The degree of $x_{0}^{k_{0}} x_{1}^{k_{1}} \in \mathrm{Jac}_{\mathbf{w}}$ is

$$
k_{0} \chi-\left(k_{0}-k_{1}\right) \chi_{1}-k_{0} \chi_{2}-\cdots-k_{0} \chi_{n+1},
$$

which is proportional to $\chi$ if and only if $k_{0}$ is even and $2 \ell-2$ divides $k_{0}-k_{1}$. Such an element can be written as

$$
\boldsymbol{a}_{k, m}:=x_{0}^{2 k+(2 \ell-2) m} x_{1}^{2 k}
$$

for $(k, m) \in\{0, \ldots, \ell-2\} \times \mathbb{N}$ which contributes $\mathbb{C}((2 k+(2 \ell-2) m) n)$ to $\mathrm{HH}^{t}$ for $t=4 k+$ $(4 \ell-6) m-(2 k+(2 \ell-2) m) n$ since $\operatorname{deg} x_{0}^{2 k} x_{1}^{2 k}=(2 k-k n) \chi$. Similarly, for each $(k, m) \in$ $\{0, \ldots, \ell-2\} \times \mathbb{N}$, there is an element $\boldsymbol{\alpha}_{k, m}$ contributing $\mathbb{C}((2 k+(2 \ell-2) m) n)$ to $\mathrm{HH}^{t}$ for $t=4 k+1+(4 \ell-6) m-(2 k+(2 \ell-2) m) n$. 


\section{Simple Singularities}

5.3.5 One has $V_{\gamma}=\mathbb{C} x_{0} \oplus \mathbb{C} x_{1} \oplus \mathbb{C} x_{2}$ if and only if $\gamma=(1,1,1,-1, \ldots,-1)$ and $n$ is odd. The degree of $x_{0}^{k_{0}} x_{1}^{k_{1}} \otimes x_{3}^{\vee} \wedge \cdots \wedge x_{n+1}^{\vee} \in \operatorname{Jac}_{\mathbf{w}_{\gamma}} \otimes \Lambda^{\operatorname{dim} N_{\gamma}} N_{\gamma}^{\vee}$ is

$$
k_{0} \chi-\left(k_{0}-k_{1}\right) \chi_{1}-k_{0} \chi_{2}-\left(k_{0}+1\right) \chi_{3}-\cdots-\left(k_{0}+1\right) \chi_{n+1},
$$

which is proportional to $\chi$ if and only if $k_{0}$ is odd and $2 \ell-2$ divides $k_{0}-k_{1}-(\ell-1)$. Such an element can be written as

$$
\boldsymbol{b}_{k, m}:=x_{0}^{k+\ell-1+(2 \ell-2) m} x_{1}^{k} \otimes x_{3}^{\vee} \wedge \cdots \wedge x_{n+1}^{\vee}
$$

for $(k, m) \in\{(k, m) \in\{0, \ldots, 2 \ell-4\} \times \mathbb{Z} \mid k+\ell$ is even and $k+\ell-1+m(2 \ell-2) \geqslant 0\}$.

It contributes $\mathbb{C}((k+\ell-1+(2 \ell-2) m) n)$ to $\mathrm{HH}^{t}$ for

$$
\begin{aligned}
t & =2 \operatorname{deg}\left(x_{0}^{k+\ell-1+(2 \ell-2) m} x_{1}^{k} \otimes x_{3}^{\vee} \wedge \cdots \wedge x_{n+1}^{\vee}\right) / \chi+\operatorname{dim} N_{\gamma} \\
& =2 k+2 \ell-3+(4 \ell-6) m-(k+\ell-1-(2 \ell-2) m) n
\end{aligned}
$$

since

$$
\begin{aligned}
\operatorname{deg} & \left(x_{0}^{k+\ell-1} x_{1}^{k} \otimes x_{3}^{\vee} \wedge \cdots \wedge x_{n+1}^{\vee}\right) \\
& =(k+\ell-1) \chi-(\ell-1) \chi_{1}-(k+\ell-1) \chi_{2}-(k+\ell) \chi_{3}-\cdots-(k+\ell) \chi_{n+1} \\
& =\left(k+\ell-1-\frac{1}{2}(k+\ell) n\right) \chi .
\end{aligned}
$$

Similarly, for each

$$
(k, m) \in\{(k, m) \in\{0, \ldots, 2 \ell-4\} \times \mathbb{Z} \mid k+\ell \text { is even and } k+\ell+m(2 \ell-2) \geqslant 0\},
$$

the element

$$
\boldsymbol{\beta}_{k, m}:=x_{0}^{\vee} \otimes x_{0}^{k+\ell+(2 \ell-2) m} x_{1}^{k} \otimes x_{3}^{\vee} \wedge \cdots \wedge x_{n+1}^{\vee} \in\left(\left(\mathbb{C} x_{0}\right)^{\vee} \otimes \mathrm{Jac}_{\mathbf{w}_{\gamma}} \otimes \Lambda^{\operatorname{dim} N_{\gamma}} N_{\gamma}^{\vee}\right)^{\Gamma}
$$

contributes $\mathbb{C}(k+\ell-1+(2 \ell-2) m)$ to $\mathrm{HH}^{t}$ for

$$
t=2 k+2 \ell-2+(4 \ell-6) m-(k+\ell-1-(2 \ell-2) m) n .
$$

5.3.6 One has $V_{\gamma}=\mathbb{C} x_{0} \oplus \mathbb{C} x_{3} \oplus \cdots \oplus \mathbb{C} x_{n+1}$ if and only if $\gamma=(1,-1,-1,1, \ldots, 1)$ with even $\ell$. An element of $\mathrm{Jac}_{\mathbf{w}_{\gamma}} \otimes \Lambda^{\operatorname{dim} N_{\gamma}} N_{\gamma}^{\vee}$ whose degree is proportional to $\chi$ can be written as

$$
\boldsymbol{c}_{m}:=x_{0}^{\ell-2+(2 \ell-2) m} \otimes x_{1}^{\vee} \wedge x_{2}^{\vee}
$$

for $m \in \mathbb{N}$, which contributes $\mathbb{C}((\ell-2+(2 \ell-2) m) n)$ to $\mathrm{HH}^{t}$ for

$$
\begin{aligned}
t & =2 \operatorname{deg}\left(x_{0}^{\ell-2} \otimes x_{1}^{\vee} \wedge x_{2}^{\vee}\right) / \chi+\operatorname{dim} N_{\gamma} \\
& =2 \ell-4+(4 \ell-6) m-(\ell-2+(2 \ell-2) m) n
\end{aligned}
$$

since $\operatorname{deg}\left(x_{0}^{\ell-2} \otimes x_{1}^{\vee} \wedge x_{2}^{\vee}\right)=\left(\ell-3-\frac{1}{2}(\ell-2) n\right) \chi$.

Similarly, for each $m \in \mathbb{N}$, there is an element $\gamma_{m}$ contributing $\mathbb{C}((\ell-2+(2 \ell-2) m) n)$ to $\mathrm{HH}^{t}$ for $t=2 \ell-3+(4 \ell-6) m-(\ell-2+(2 \ell-2) m) n$.

5.3.7 One has $V_{\gamma}=\mathbb{C} x_{0}$ if and only if $\ell$ is even, $n$ is odd, and $\gamma=(1,-1, \ldots,-1) \in$ ker $\chi$. The degree of

$$
x_{0}^{k_{0}} \otimes x_{1}^{\vee} \wedge \cdots \wedge x_{n+1}^{\vee} \in \mathrm{Jac}_{\mathbf{w}_{\gamma}} \otimes \Lambda^{\operatorname{dim} N_{\gamma}} N_{\gamma}^{\vee}
$$

is given by

$$
k_{0} \chi-\left(k_{0}+1\right) \chi_{1}-\left(k_{0}+1\right) \chi_{2}-\cdots-\left(k_{0}+1\right) \chi_{n+1},
$$




\section{Y. LEKILI AND K. UEDA}

which is proportional to $\chi$ if and only if $2 \ell-2$ divides $k_{0}+1$. Such an element can be written as

$$
\boldsymbol{d}_{m}:=x_{0}^{-1+m(2 \ell-2)} \otimes x_{1}^{\vee} \wedge \cdots \wedge x_{n+1}^{\vee}
$$

for $m \in \mathbb{N} \backslash\{0\}$. Since $\operatorname{deg}\left(x_{0}^{-1} \otimes x_{1}^{\vee} \wedge \cdots \wedge x_{n+1}^{\vee}\right)=-\chi$, each such element contributes $\mathbb{C}((-1+$ $(2 \ell-2) m) n)$ to $\mathrm{HH}^{t}$ for

$$
\begin{aligned}
t & =2 \operatorname{deg}\left(x_{0}^{-1+(2 \ell-2) m} \otimes x_{1}^{\vee} \wedge \cdots \wedge x_{n+1}^{\vee}\right) / \chi+\operatorname{dim} N_{\gamma} \\
& =-1+(4 \ell-6) m-(-1+(2 \ell-2) m) n .
\end{aligned}
$$

Similarly, for each $m \in \mathbb{N}$, the element

$$
\boldsymbol{\delta}_{m}:=x_{0}^{\vee} \otimes x_{0}^{m(2 \ell-2)} \otimes x_{1}^{\vee} \wedge \cdots \wedge x_{n+1}^{\vee} \in \mathbb{C} x_{0}^{\vee} \otimes \mathrm{Jac}_{\mathbf{w}_{\gamma}} \otimes \Lambda^{\operatorname{dim} N_{\gamma}} N_{\gamma}^{\vee}
$$

contributes $\mathbb{C}((-1+(2 \ell-2) m) n)$ to $\mathrm{HH}^{t}$ for $t=(4 \ell-6) m-(-1+(2 \ell-2) m) n$.

5.3.8 One has $V_{\gamma}=\mathbb{C} x_{0} \oplus \mathbb{C} x_{2}$ if and only if $\ell$ is odd, $n$ is even, and $\gamma=(1,-1,1,-1, \ldots,-1)$. The degree of

$$
x_{0}^{k_{0}} \otimes x_{1}^{\vee} \wedge x_{3}^{\vee} \wedge \cdots \wedge x_{n+1}^{\vee} \in \mathrm{Jac}_{\mathbf{w}_{\gamma}} \otimes \Lambda^{\operatorname{dim} N_{\gamma}} N_{\gamma}^{\vee}
$$

is given by

$$
k_{0} \chi_{0}-\chi_{1}-\chi_{3}-\cdots-\chi_{n+1}=\left(k_{0}(\ell-2)-1\right) \chi_{1}-\left(k_{0}+1\right) \chi_{3}-\cdots-\left(k_{0}+1\right) \chi_{n+1},
$$

which is proportional to $\chi$ if and only if $k_{0}$ is odd and $2 \ell-2$ divides $k_{0}(\ell-2)-1$. Such an element can be written as

$$
\boldsymbol{e}_{m}:=x_{0}^{\ell-2+(2 \ell-2) m} \otimes x_{1}^{\vee} \wedge x_{3}^{\vee} \wedge \cdots \wedge x_{n+1}^{\vee}
$$

for $m \in \mathbb{N}$, which contributes $\mathbb{C}((\ell-2+(2 \ell-2) m) n)$ to $\mathrm{HH}^{t}$ for

$$
t=2 \ell-4+(4 \ell-6) m-(\ell-2+(2 \ell-2) m) n
$$

since $\operatorname{deg}\left(x_{0}^{\ell-2} \otimes x_{1}^{\vee} \wedge x_{3}^{\vee} \wedge \cdots \wedge x_{n+1}^{\vee}\right)=\frac{1}{2}(2 \ell-4-(\ell-1) n) \chi$.

Similarly, for each $m \in \mathbb{N}$, there is an element $\boldsymbol{\epsilon}_{m}$ contributing $\mathbb{C}((\ell-2+(2 \ell-2) m) n)$ to $\mathrm{HH}^{t}$ for $t=2 \ell-3+(4 \ell-6) m-(\ell-2+(2 \ell-2) m) n$.

5.3.9 Now, we move on to the case when $\mathbb{C} x_{0} \not \subset V_{\gamma}$. We divide it into three cases:

$$
\mathbb{C} x_{1} \subset V_{\gamma}, \quad \mathbb{C} x_{1} \not \subset V_{\gamma} \text { and } V_{\gamma} \neq 0, \quad V_{\gamma}=0 .
$$

5.3.10 Set $\zeta:=\exp (2 \pi \sqrt{-1} /(2 \ell-2))$. For a given $\gamma=\left(t_{0}, \ldots, t_{n+1}\right) \in$ ker $\chi$, we write $t_{1}=\zeta^{p}$ for $p \in\{0, \ldots, 2 \ell-3\}$. Then one has $t_{2}=(-1)^{p}$, so that

- $V_{\gamma}$ contains $\mathbb{C} x_{1}$ if and only if $p=0$, and

- $V_{\gamma}$ contains $\mathbb{C} x_{2}$ if and only if $p$ is even.

5.3.11 If $\mathbb{C} x_{0} \not \subset V_{\gamma}$ and $\mathbb{C} x_{1} \subset V_{\gamma}$, then one has that $\gamma=(-1,1,1,-1, \ldots,-1), n$ is even, and $V_{\gamma}=\mathbb{C} x_{1} \oplus \mathbb{C} x_{2}$. The element

$$
x_{1}^{\ell-2} \otimes x_{0}^{\vee} \wedge x_{3}^{\vee} \wedge \cdots \wedge x_{n+1}^{\vee}
$$

has degree $(\ell-2) \chi_{1}-\chi_{0}-\chi_{3}-\cdots-\chi_{n+1}=0$, so that it contributes $\mathbb{C}(-n)$ to $\mathrm{HH}^{t}$ for $t=\operatorname{dim} N_{\gamma}=n$, and this is the only contribution. 


\section{SiMPLE SINGULARITIES}

5.3.12 If $\mathbb{C} x_{0} \not \subset V_{\gamma}, \mathbb{C} x_{1} \not \subset V_{\gamma}$, and $V_{\gamma} \neq 0$, then $V_{\gamma}$ is either $V_{\gamma}=\mathbb{C} x_{2}, \mathbb{C} x_{2} \oplus \cdots \oplus \mathbb{C} x_{n+1}$ or $\mathbb{C} x_{3} \oplus \cdots \oplus \mathbb{C} x_{n+1}$. No such $\gamma$ contributes to $\mathrm{HH}^{*}$ since $\mathrm{Jac}_{\mathbf{w}_{\gamma}} \otimes \Lambda^{\operatorname{dim} N_{\gamma}} N_{\gamma}^{\vee}$ is spanned by a single element whose degree is not proportional to $\chi$.

5.3.13 One has $V_{\gamma}=0$ if and only if $t_{3}=\cdots=t_{n+1}=-1, t_{1}=\zeta^{2 m+1}$ for $m \in\{0, \ldots, \ell-2\}$ and $t_{0}=(-1)^{n} \zeta^{-2 m-1} \neq 1$. The number of such $\gamma$ is $\ell-2$ if $\ell$ is even and $n$ is odd, and $\ell-1$ otherwise. Each such $\gamma$ contributes $\mathbb{C}(-n)$ to $\mathrm{HH}^{n}$.

5.3.14 To sum up, the Hochschild cohomology group has a basis consisting of the following elements:

- $\boldsymbol{a}_{k, m}$ of degree $4 k+(4 \ell-6) m-(2 k+(2 \ell-2) m) n$ and weight $-(2 k+(2 \ell-2) m) n$ for $(k, m) \in\{0, \ldots, \ell-2\} \times \mathbb{N}$,

- $\boldsymbol{\alpha}_{k, m}$ of degree $4 k+1+(4 \ell-6) m-(2 k+(2 \ell-2) m) n$ and weight $-(2 k+(2 \ell-2) m) n$ for $(k, m) \in\{0, \ldots, \ell-2\} \times \mathbb{N}$,

- if $n$ is odd, $\boldsymbol{b}_{k, m}$ of degree $2 k+2 \ell-3+(4 \ell-6) m-(k+\ell-1-(2 \ell-2) m) n$ and weight $-(k+\ell-1+(2 \ell-2) m) n$ for $\{(k, m) \in\{0, \ldots, 2 \ell-4\} \times \mathbb{Z} \mid k+\ell$ is even and $k+\ell-1+$ $m(2 \ell-2) \geqslant 0\}$,

- if $n$ is odd, $\boldsymbol{\beta}_{k, m}$ of degree $2 k+2 \ell-2+(4 \ell-6) m-(k+\ell-1-(2 \ell-2) m) n$ and weight $-(k+\ell-$ $1-(2 \ell-2) m) n$ for $\{(k, m) \in\{0, \ldots, 2 \ell-4\} \times \mathbb{Z} \mid k+\ell$ is even and $k+\ell+m(2 \ell-2) \geqslant 0\}$,

- if $\ell$ is even, $\boldsymbol{c}_{m}$ of degree $2 \ell-4+(4 \ell-6) m-(\ell-2+(2 \ell-2) m) n$ and weight $-(\ell-2+(2 \ell-2) m) n$ for $m \in \mathbb{N}$,

- if $\ell$ is even, $\gamma_{m}$ of degree $2 \ell-3+(4 \ell-6) m-(\ell-2+(2 \ell-2) m) n$ and weight $-(\ell-2+(2 \ell-2) m) n$ for $m \in \mathbb{N}$,

- if $\ell$ is even and $n$ is odd, $\boldsymbol{d}_{m}$ of degree $-1+(4 \ell-6) m-(-1+(2 \ell-2) m) n$ and weight $-(-1+(2 \ell-2) m) n$ for $m \in \mathbb{N} \backslash\{0\}$,

- if $\ell$ is even and $n$ is odd, $\boldsymbol{\delta}_{m}$ of degree $(4 \ell-6) m-(-1+(2 \ell-2) m) n$ and weight $-(-1+$ $(2 \ell-2) m) n$ for $m \in \mathbb{N}$,

- if $\ell$ is odd and $n$ is even, $\boldsymbol{e}_{m}$ of degree $2 \ell-4+(4 \ell-6) m-(\ell-2+(2 \ell-2) m) n$ and weight $-(\ell-2+(2 \ell-2) m) n$ for $m \in \mathbb{N}$,

- if $\ell$ is odd and $n$ is even, $\boldsymbol{\epsilon}_{m}$ of degree $2 \ell-3+(4 \ell-6) m-(\ell-2+(2 \ell-2) m) n$ and weight $-(\ell-2+(2 \ell-2) m) n$ for $m \in \mathbb{N}$, and

- $\boldsymbol{s}_{h}$ of degree $n$ and weight $n$, where $h$ runs over a set consisting of

$* \ell-2$ elements if $\ell$ is even and $n$ is odd,

$* \ell-1$ elements if both $\ell$ and $n$ are odd, and

$* \ell$ elements otherwise.

\subsection{Type $E_{6}$}

Consider the case

$$
\begin{gathered}
\mathbf{w}=x_{1}^{4}+x_{2}^{3}+x_{3}^{2}+\cdots+x_{n+1}^{2} \in \mathbb{C}\left[x_{0}, x_{1}, \ldots, x_{n+1}\right], \\
\Gamma=\Gamma_{\mathbf{w}}:=\left\{\gamma=\left(t_{0}, t_{1}, \ldots, t_{n+1}\right) \in\left(\mathbb{G}_{\mathrm{m}}\right)^{n+2} \mid t_{1}^{4}=t_{2}^{3}=t_{3}^{2}=\cdots=t_{n+1}^{2}=t_{0} t_{1} \cdots t_{n+1}\right\},
\end{gathered}
$$




\section{Y. LEKILI AND K. UEDA}

so that $\operatorname{ker} \chi \cong \boldsymbol{\mu}_{4} \times \boldsymbol{\mu}_{3} \times\left(\boldsymbol{\mu}_{2}\right)^{n-1}$ and $\operatorname{Char}(\Gamma)$ is generated by $\chi$ and $\chi_{i}=\operatorname{deg} x_{i}$ for $i \in$ $\{0, \ldots, n+1\}$ with relations

$$
\chi=4 \chi_{1}=3 \chi_{2}=2 \chi_{3}=\cdots=2 \chi_{n+1}=\chi_{0}+\cdots+\chi_{n+1} .
$$

5.4.1 For any $\gamma \in$ ker $\chi$, one has

$$
\mathrm{Jac}_{\mathbf{w}_{\gamma}} \cong\left\{\begin{array} { l l } 
{ \mathbb { C } [ x _ { 0 } ] , } & { \mathbb { C } x _ { 0 } \subset V _ { \gamma } } \\
{ \mathbb { C } , } & { \mathbb { C } x _ { 0 } \not \subset V _ { \gamma } }
\end{array} \otimes \left\{\begin{array} { l l } 
{ \mathbb { C } [ x _ { 1 } ] / ( x _ { 1 } ^ { 3 } ) , } & { \mathbb { C } x _ { 1 } \subset V _ { \gamma } } \\
{ \mathbb { C } , } & { \mathbb { C } x _ { 1 } \not \subset V _ { \gamma } }
\end{array} \otimes \left\{\begin{array}{ll}
\mathbb{C}\left[x_{2}\right] /\left(x_{2}^{2}\right), & \mathbb{C} x_{2} \subset V_{\gamma}, \\
\mathbb{C}, & \mathbb{C} x_{2} \not \subset V_{\gamma} .
\end{array}\right.\right.\right.
$$

If we write an element of $\mathrm{Jac}_{\mathbf{w}_{\gamma}} \otimes \Lambda^{\operatorname{dim} N_{\gamma}} N_{\gamma}^{\vee}$ as $x_{0}^{k_{0}} x_{1}^{k_{1}} x_{2}^{k_{2}} \otimes x_{j_{1}}^{\vee} \wedge x_{j_{2}}^{\vee} \wedge \cdots \wedge x_{j_{s}}^{\vee}$, where $k_{i}=0$ if $\mathbb{C} x_{i} \not \subset V_{\gamma}$ for $i=0,1,2$, then its degree is given by

$$
k_{0} \chi_{0}+k_{1} \chi_{1}+k_{2} \chi_{2}-\chi_{j_{1}}-\cdots-\chi_{j_{s}},
$$

which can be proportional to $\chi$ only if $V_{\gamma} \cap\left(\mathbb{C} x_{3} \oplus \cdots \oplus \mathbb{C} x_{n+1}\right)$ is either $\mathbb{C} x_{3} \oplus \cdots \oplus \mathbb{C} x_{n+1}$ or 0 . We will assume this condition for the rest of Section 5.4 and divide the analysis into the following three cases:

$$
\mathbb{C} x_{0} \subset V_{\gamma}, \quad \mathbb{C} x_{0} \not \subset V_{\gamma} \text { and } V_{\gamma} \neq 0, \quad V_{\gamma}=0 .
$$

5.4.2 Since $t_{0}=1$ implies $t_{2}=1$ and $t_{1}= \pm 1$, one has the following:

- $V_{\gamma}$ contains $\mathbb{C} x_{0}$ if and only if

$* \gamma=(1, \ldots, 1)$, where $V_{\gamma}=V$;

$* \gamma=(1,1,1,-1, \ldots,-1)$ with odd $n \geqslant 3$, where $V_{\gamma}=\mathbb{C} x_{0} \oplus \mathbb{C} x_{1} \oplus \mathbb{C} x_{2}$; or

$* \gamma=(1,-1,1,-1, \ldots,-1)$ with even $n$, where $V_{\gamma}=\mathbb{C} x_{0} \oplus \mathbb{C} x_{2}$.

5.4.3 One has $V_{\gamma}=V$ if and only if $\gamma$ is the identity element. The degree of $x_{0}^{k_{0}} x_{1}^{k_{1}} x_{2}^{k_{2}} \in$ $\mathrm{Jac}_{\mathrm{w}}$ is

$$
k_{0} \chi-\left(k_{0}-k_{1}\right) \chi_{1}-\left(k_{0}-k_{2}\right) \chi_{2}-k_{0} \chi_{3}-\cdots-k_{0} \chi_{n+1},
$$

which is proportional to $\chi$ if and only if

- 4 divides $k_{0}-k_{1}$ and 3 divides $k_{0}-k_{2}$ if $n=1$, and

- 4 divides $k_{0}-k_{1}, 3$ divides $k_{0}-k_{2}$, and $k_{0}$ be even if $n>1$.

Thus, for $n=1$, we must have

$$
5 k_{0}+3 k_{1}+4 k_{2}=12 m
$$

for $m \in \mathbb{N}$, in which case one has $\operatorname{deg} x_{0}^{k_{0}} x_{1}^{k_{1}} x_{2}^{k_{2}}=m \chi$.

For each $m \in \mathbb{N}$ such that $5 \nmid m$, the equation (5.10) has a unique solution with $\left(k_{1}, k_{2}\right) \in$ $\{0,1,2\} \times\{0,1\}$, and if $5 \mid m$, then there are precisely two contributions with $\left(k_{1}, k_{2}\right)=(0,0)$ and $\left(k_{1}, k_{2}\right)=(2,1)$ such that $\left(k_{1}, k_{2}, m\right) \in\{0,1,2\} \times\{0,1\} \times \mathbb{N}$ except if $m=0$, in which case only $\left(k_{1}, k_{2}\right)=(0,0)$ contributes. Each such $\left(k_{1}, k_{2}, m\right)$ contributes $\mathbb{C}\left(k_{0} n\right)$ to $\mathrm{HH}^{t}$ and $\mathrm{HH}^{t+1}$ for $t=2 m$.

For $n>1$, the condition that $k_{0}$ is even forces $k_{1} \neq 1$, and the possible $\left(k_{0}, k_{1}, k_{2}\right)$ and 


\section{SimPle SINGULARITIES}

$t=2 \operatorname{deg}\left(x_{0}^{k_{0}} x_{1}^{k_{1}} x_{2}^{k_{2}}\right) / \chi$ are given by

\begin{tabular}{ccc}
\hline$\left(k_{1}, k_{2}\right)$ & $k_{0}$ & $t$ \\
\hline$(0,0)$ & $12 m$ & $22 m-12 m n$ \\
$(0,1)$ & $4+12 m$ & $8+22 m-(4+12 m) n$ \\
$(2,0)$ & $6+12 m$ & $12+22 m-(6+12 m) n$ \\
$(2,1)$ & $10+12 m$ & $20+22 m-(10+12 m) n$ \\
\hline
\end{tabular}

for $m \in \mathbb{N}$. Each $\left(k_{0}, k_{1}, k_{2}\right)$ from $(5.11)$ contributes $\mathbb{C}\left(k_{0} n\right)$ to $\mathrm{HH}^{t}$ and $\mathrm{HH}^{t+1}$.

5.4.4 One has $V_{\gamma}=\mathbb{C} x_{0} \oplus \mathbb{C} x_{1} \oplus \mathbb{C} x_{2} \subsetneq V$ for $\gamma \in$ ker $\chi$ if and only if $n$ is an odd integer greater than or equal to 3 and $\gamma=(1,1,1,-1, \ldots,-1)$. The degree of

$$
x_{0}^{k_{0}} x_{1}^{k_{1}} x_{2}^{k_{2}} \otimes x_{3}^{\vee} \wedge \cdots \wedge x_{n+1}^{\vee} \in \operatorname{Jac}_{\mathbf{w}_{\gamma}} \otimes \Lambda^{\operatorname{dim} N_{\gamma}} N_{\gamma}^{\vee}
$$

is given by

$$
k_{0} \chi-\left(k_{0}-k_{1}\right) \chi_{1}-\left(k_{0}-k_{2}\right) \chi_{2}-\left(k_{0}+1\right) \chi_{3}-\cdots-\left(k_{0}+1\right) \chi_{n+1},
$$

which is proportional to $\chi$ if and only if $k_{0}$ is odd, 4 divides $k_{0}-k_{1}$, and 3 divides $k_{0}-k_{2}$. This forces $k_{1}=1$, and the possible $\left(k_{0}, k_{1}, k_{2}\right)$ and

$$
t=2 \operatorname{deg}\left(x_{0}^{k_{0}} x_{1}^{k_{1}} x_{2}^{k_{2}} \otimes x_{3}^{\vee} \wedge \cdots \wedge x_{n+1}^{\vee}\right) / \chi+\operatorname{dim} N_{\gamma}
$$

are given by

\begin{tabular}{ccc}
\hline$\left(k_{1}, k_{2}\right)$ & $k_{0}$ & $t$ \\
\hline$(1,0)$ & $9+12 m$ & $17+22 m-(9+12 m) n$ \\
$(1,1)$ & $1+12 m$ & $3+22 m-(1+12 m) n$ \\
\hline
\end{tabular}

for $m \in \mathbb{N}$. Each $\left(k_{0}, k_{1}, k_{2}\right)$ from $(5.12)$ contributes $\mathbb{C}\left(k_{0} n\right)$ to $\mathrm{HH}^{t}$ and $\mathrm{HH}^{t+1}$.

5.4.5 One has $V_{\gamma}=\mathbb{C} x_{0} \oplus \mathbb{C} x_{2}$ if and only if $n$ is even and $\gamma=(1,-1,1,-1, \ldots,-1) \in$ ker $\chi$. The degree of

$$
x_{0}^{k_{0}} x_{2}^{k_{2}} \otimes x_{1}^{\vee} \wedge x_{3}^{\vee} \wedge \cdots \wedge x_{n+1}^{\vee} \in \mathrm{Jac}_{\mathbf{w}_{\gamma}} \otimes \Lambda^{\operatorname{dim} N_{\gamma}} N_{\gamma}^{\vee}
$$

is given by

$$
k_{0} \chi-\left(k_{0}+1\right) \chi_{1}-\left(k_{0}-k_{2}\right) \chi_{2}-\left(k_{0}+1\right) \chi_{3}-\cdots-\left(k_{0}+1\right) \chi_{n+1},
$$

which is proportional to $\chi$ if and only if 4 divides $k_{0}+1$ and 3 divides $k_{0}-k_{2}$. The possible $\left(k_{0}, k_{2}\right)$ and

$$
t=2 \operatorname{deg}\left(x_{0}^{k_{0}} x_{2}^{k_{2}} \otimes x_{1}^{\vee} \wedge x_{3}^{\vee} \wedge \cdots \wedge x_{n}^{\vee}\right) / \chi+\operatorname{dim} N_{\gamma}
$$

are given by

\begin{tabular}{ccc}
\hline$k_{2}$ & $k_{0}$ & $t$ \\
\hline 0 & $3+12 m$ & $6+22 m-(3+12 m) n$ \\
1 & $7+12 m$ & $14+22 m-(7+12 m) n$ \\
\hline
\end{tabular}

for $m \in \mathbb{N}$. Each $\left(k_{0}, k_{2}\right)$ from $(5.13)$ contributes $\mathbb{C}\left(k_{0} n\right)$ to $\mathrm{HH}^{t}$ and $\mathrm{HH}^{t+1}$. 


\section{Y. LEKILI AND K. UEDA}

5.4.6 If $V_{\gamma}=\mathbb{C} x_{1}$, then one has

$\operatorname{deg}\left(x_{1}^{k_{1}} \otimes x_{0}^{\vee} \wedge x_{2}^{\vee} \wedge \cdots \wedge x_{n+1}^{\vee}\right)=-\chi_{0}+k_{1} \chi_{1}-\chi_{2}-\cdots-\chi_{n+1}=-\chi+\left(k_{1}+1\right) \chi_{1}$,

which is not proportional to $\chi$ for any $k_{1} \in\{0,1,2\}$. Similarly, $\gamma$ with $\mathbb{C} x_{0} \not \subset V_{\gamma}$ and $V_{\gamma} \neq 0$ does not contribute to $\mathrm{HH}^{*}$.

5.4.7 One has $V_{\gamma}=0$ if and only if $t_{1} \in\left(\boldsymbol{\mu}_{4} \backslash\{1\}\right), t_{2} \in\left(\boldsymbol{\mu}_{3} \backslash\{1\}\right)$, and $t_{3}=\cdots, t_{n+1}=-1$, since $t_{2} \neq 1$ implies $t_{0}=(-1)^{n-1} t_{1}^{-1} t_{2}^{-1} \neq 1$. There are six such $\gamma$, and each of them contributes $\mathbb{C}(-n)$ to $\mathrm{HH}^{n}$.

\subsection{Type $E_{7}$}

Consider the case

$$
\begin{gathered}
\mathbf{w}=x_{1}^{3} x_{2}+x_{2}^{3}+x_{3}^{2}+\cdots+x_{n+1}^{2} \in \mathbb{C}\left[x_{0}, x_{1}, \ldots, x_{n+1}\right], \\
\Gamma=\Gamma_{\mathbf{w}}:=\left\{\gamma=\left(t_{0}, \ldots, t_{n+1}\right) \in\left(\mathbb{G}_{\mathrm{m}}\right)^{n+2} \mid t_{1}^{3} t_{2}=t_{2}^{3}=t_{3}^{2}=\cdots=t_{n+1}^{2}=t_{0} \cdots t_{n+1}\right\},
\end{gathered}
$$

so that ker $\chi \cong \boldsymbol{\mu}_{9} \times\left(\boldsymbol{\mu}_{2}\right)^{n-1}$ and $\operatorname{Char}(\Gamma)$ is generated by $\chi$ and $\chi_{i}=\operatorname{deg} x_{i}$ for $i \in\{0, \ldots, n+1\}$ with relations

$$
\chi=3 \chi_{1}+\chi_{2}=3 \chi_{2}=2 \chi_{3}=\cdots=2 \chi_{n+1}=\chi_{0}+\cdots+\chi_{n+1} .
$$

These relations imply

$$
\begin{gathered}
\chi_{2}=\chi-3 \chi_{1}, \quad 9 \chi_{1}=2 \chi \\
\chi_{0}=\chi-\chi_{1}-\cdots-\chi_{n+1}=2 \chi_{1}-\chi_{3}-\cdots-\chi_{n+1} .
\end{gathered}
$$

5.5.1 For any $\gamma \in$ ker $\chi$, the intersection $V_{\gamma} \cap\left(\mathbb{C} x_{1} \oplus \mathbb{C} x_{2}\right)$ can be $\mathbb{C} x_{1} \oplus \mathbb{C} x_{2}, \mathbb{C} x_{2}$, or 0 , where $\mathrm{Jac}_{\mathbf{w}_{\gamma}^{\prime}}$ is isomorphic to $\mathbb{C}\left[x_{1}, x_{2}\right] /\left(3 x_{1}^{2} x_{2}, x_{1}^{3}+3 x_{2}^{2}\right), \mathbb{C}\left[x_{2}\right] /\left(3 x_{2}^{2}\right)$, or $\mathbb{C}$, respectively. A basis of $\mathbb{C}\left[x_{1}, x_{2}\right] /\left(3 x_{1}^{2} x_{2}, x_{1}^{3}+3 x_{2}^{2}\right)$ is given by $\left\{1, x_{1}, x_{1}^{2}, x_{1}^{3}, x_{1}^{4}, x_{2}, x_{1} x_{2}\right\}$.

If we write an element of $\mathrm{Jac}_{\mathbf{w}_{\gamma}} \otimes \Lambda^{\operatorname{dim} N_{\gamma}} N_{\gamma}^{\vee}$ as $x_{0}^{k_{0}} x_{1}^{k_{1}} x_{2}^{k_{2}} \otimes x_{j_{1}}^{\vee} \wedge x_{j_{2}}^{\vee} \wedge \cdots \wedge x_{j_{s}}^{\vee}$, then its degree is given by

$$
k_{0} \chi_{0}+k_{1} \chi_{1}+k_{2} \chi_{2}-\chi_{j_{1}}-\cdots-\chi_{j_{s}},
$$

which can be proportional to $\chi$ only if $V \cap\left(\mathbb{C} x_{3} \oplus \cdots \oplus \mathbb{C} x_{n+1}\right)$ is either $\mathbb{C} x_{3} \oplus \cdots \oplus \mathbb{C} x_{n+1}$ or 0 . We assume this condition for the rest of Section 5.5.

5.5.2 For $\gamma=\left(t_{0}, \ldots, t_{n+1}\right) \in \operatorname{ker} \chi$, one has $t_{1}^{2}=t_{0} t_{3} \cdots t_{n+1}= \pm t_{0}$ and $t_{2}^{2}=t_{0} t_{1} t_{3} \cdots t_{n+1}$ $= \pm t_{0} t_{1}$, so that the condition $t_{0}=1$ implies $t_{1}^{2}= \pm 1$ and $t_{2}^{2}= \pm t_{1}$, which together with $t_{2}^{3}=1$ imply $t_{1}=t_{2}=1$. Hence, one has $\mathbb{C} x_{0} \subset V_{\gamma}$ if and only if either $V_{\gamma}=V$ or $V_{\gamma}=\mathbb{C} x_{0} \oplus \mathbb{C} x_{1} \oplus \mathbb{C} x_{2}$.

5.5.3 One has $V_{\gamma}=V$ if and only if $\gamma$ is the identity element. The degree of $x_{0}^{k_{0}} x_{1}^{k_{1}} x_{2}^{k_{2}} \in$ $\mathrm{Jac}_{\mathbf{w}}$ is

$$
\begin{aligned}
& k_{0}\left(2 \chi_{1}-\chi_{3}-\cdots-\chi_{n+1}\right)+k_{1} \chi_{1}+k_{2}\left(\chi-3 \chi_{1}\right) \\
& \quad=k_{2} \chi+\left(2 k_{0}+k_{1}-3 k_{2}\right) \chi_{1}-k_{0} \chi_{3}-\cdots-k_{0} \chi_{n+1},
\end{aligned}
$$

which is proportional to $\chi$ if and only if

- 9 divides $2 k_{0}+k_{1}-3 k_{2}$ if $n=1$, and

- 9 divides $2 k_{0}+k_{1}-3 k_{2}$ and $k_{0}$ is even if $n>1$. 


\section{SiMPLE SINGULARITIES}

For $n=1$, one has

$$
t:=2 \operatorname{deg}\left(x_{0}^{k_{0}} x_{1}^{k_{1}} x_{2}^{k_{2}}\right) / \chi=2 k_{2}+\frac{4}{9}\left(2 k_{0}+k_{1}-3 k_{2}\right) .
$$

The possible $\left(k_{0}, k_{1}, k_{2}\right)$ and $t$ are given by

\begin{tabular}{ccc}
\hline$\left(k_{1}, k_{2}\right)$ & $k_{0}$ & $t$ \\
\hline$(0,0)$ & $9 m$ & $8 m$ \\
$(1,0)$ & $4+9 m$ & $4+8 m$ \\
$(2,0)$ & $8+9 m$ & $8+8 m$ \\
$(3,0)$ & $3+9 m$ & $4+8 m$ \\
$(4,0)$ & $7+9 m$ & $8+8 m$ \\
$(0,1)$ & $6+9 m$ & $6+8 m$ \\
$(1,1)$ & $1+9 m$ & $2+8 m$ \\
\hline
\end{tabular}

for $m \in \mathbb{N}$. Each $\left(k_{0}, k_{1}, k_{2}\right)$ from $(5.14)$ contributes $\mathbb{C}\left(k_{0} n\right)$ to $\mathrm{HH}^{t}$ and $\mathrm{HH}^{t+1}$.

In addition, for the case $\left(k_{1}, k_{2}\right)=(2,0)$, the element $x_{0}^{\vee} \otimes x_{1}^{2}$ corresponding to $m=-1$ in (5.14) has degree 0 and contributes $\mathbb{C}(-1)$ to $\mathrm{HH}^{1}$.

For $n>1$, one has

$$
t:=2 \operatorname{deg}\left(x_{0}^{k_{0}} x_{1}^{k_{1}} x_{2}^{k_{2}}\right) / \chi=2 k_{2}+\frac{4}{9}\left(2 k_{0}+k_{1}-3 k_{2}\right)-k_{0}(n-1) .
$$

The possible $\left(k_{0}, k_{1}, k_{2}\right)$ and $t$ are given by

\begin{tabular}{ccc}
\hline$\left(k_{1}, k_{2}\right)$ & $k_{0}$ & $t$ \\
\hline$(0,0)$ & $18 m$ & $34 m-18 m n$ \\
$(1,0)$ & $4+18 m$ & $8+34 m-(4+18 m) n$ \\
$(2,0)$ & $8+18 m$ & $16+34 m-(8+18 m) n$ \\
$(3,0)$ & $12+18 m$ & $24+34 m-(12+18 m) n$ \\
$(4,0)$ & $16+18 m$ & $32+34 m-(16+18 m) n$ \\
$(0,1)$ & $6+18 m$ & $12+34 m-(6+18 m) n$ \\
$(1,1)$ & $10+18 m$ & $20+34 m-(10+18 m) n$ \\
\hline
\end{tabular}

for $m \in \mathbb{N}$. Each $\left(k_{0}, k_{1}, k_{2}\right)$ from $(5.15)$ contributes $\mathbb{C}\left(k_{0} n\right)$ to $\mathrm{HH}^{t}$ and $\mathrm{HH}^{t+1}$.

5.5.4 For $n>1$, in addition, one has $V_{\gamma}=\mathbb{C} x_{0} \oplus \mathbb{C} x_{1} \oplus \mathbb{C} x_{2}$ if and only if $n$ is odd and $\gamma=(1,1,1,-1, \ldots,-1)$. The degree of

$$
x_{0}^{k_{0}} x_{1}^{k_{1}} x_{2}^{k_{2}} \otimes x_{3}^{\vee} \wedge \cdots \wedge x_{n+1}^{\vee} \in \mathrm{Jac}_{\mathbf{w}_{\gamma}} \otimes \Lambda^{\operatorname{dim} N_{\gamma}} N_{\gamma}^{\vee}
$$

is given by

$$
\begin{aligned}
& k_{0}\left(2 \chi_{1}-\chi_{3}-\cdots-\chi_{n+1}\right)+k_{1} \chi_{1}+k_{2}\left(\chi-3 \chi_{1}\right)-\chi_{3}-\cdots-\chi_{n+1} \\
& \quad=k_{2} \chi+\left(2 k_{0}+k_{1}-3 k_{2}\right) \chi_{1}-\left(k_{0}+1\right) \chi_{3}-\cdots-\left(k_{0}+1\right) \chi_{n+1},
\end{aligned}
$$

which is proportional to $\chi$ if and only if 9 divides $2 k_{0}+k_{1}-3 k_{2}$ and $k_{0}$ is odd. The possible $\left(k_{0}, k_{1}, k_{2}\right)$ and

$$
t:=2 \operatorname{deg}\left(x_{0}^{k_{0}} x_{1}^{k_{1}} x_{2}^{k_{2}} \otimes x_{3}^{\vee} \wedge \cdots \wedge x_{n+1}^{\vee}\right) / \chi+\operatorname{dim} N_{\gamma}
$$


are given by

\begin{tabular}{ccc}
\hline$\left(k_{1}, k_{2}\right)$ & $k_{0}$ & $t$ \\
\hline$(0,0)$ & $9+18 m$ & $17+34 m-(9+18 m) n$ \\
$(1,0)$ & $13+18 m$ & $25+34 m-(13+18 m) n$ \\
$(2,0)$ & $17+18 m$ & $33+34 m-(17+18 m) n$ \\
$(3,0)$ & $3+18 m$ & $7+34 m-(3+18 m) n$ \\
$(4,0)$ & $7+18 m$ & $15+34 m-(7+18 m) n$ \\
$(0,1)$ & $15+18 m$ & $29+34 m-(15+18 m) n$ \\
$(1,1)$ & $1+18 m$ & $3+34 m-(1+18 m) n$ \\
\hline
\end{tabular}

for $m \in \mathbb{N}$. Each $\left(k_{0}, k_{1}, k_{2}\right)$ from $(5.16)$ contributes $\mathbb{C}\left(k_{0} n\right)$ to $\mathrm{HH}^{t}$ and $\mathrm{HH}^{t+1}$.

In addition, for the case $\left(k_{1}, k_{2}\right)=(2,0)$, the element $x_{0}^{\vee} \otimes x_{1}^{2} \otimes x_{3}^{\vee} \wedge \cdots \wedge x_{n+1}^{\vee}$ corresponding to $m=-1$ in (5.16) has degree 0 and contributes $\mathbb{C}(-n)$ to $\mathrm{HH}^{n}$.

5.5.5 One has $V_{\gamma}=0$ for $\gamma=\left(t_{0}, \ldots, t_{n+1}\right) \in \operatorname{ker} \chi$ if and only if $t_{1} \in \boldsymbol{\mu}_{9} \backslash\{1\}, t_{2}:=t_{1}^{-3} \neq 1$, and $t_{3}=\cdots=t_{n+1}=-1$, in which case one has $t_{0}=(-1)^{n-1} t_{1}^{2} \neq 1$. The set $\left\{t_{1} \in \boldsymbol{\mu}_{9} \mid t_{1}^{3} \neq 1\right\}$ consists of six elements, each of which contributes $\mathbb{C}(-n)$ to $\mathrm{HH}^{n}$.

\subsection{Type $E_{8}$}

Consider the case

$$
\begin{gathered}
\mathbf{w}=x_{1}^{5}+x_{2}^{3}+x_{3}^{2}+\cdots+x_{n+1}^{2} \in \mathbb{C}\left[x_{0}, x_{1}, \ldots, x_{n+1}\right] \\
\Gamma=\Gamma_{\mathbf{w}}:=\left\{\gamma=\left(t_{0}, \ldots, t_{n+1}\right) \in\left(\mathbb{G}_{\mathrm{m}}\right)^{n+2} \mid t_{1}^{5}=t_{2}^{3}=t_{3}^{2}=\cdots=t_{n+1}^{2}=t_{0} \cdots t_{n+1}\right\},
\end{gathered}
$$

so that $\operatorname{ker} \chi \cong \boldsymbol{\mu}_{5} \times \boldsymbol{\mu}_{3} \times\left(\boldsymbol{\mu}_{2}\right)^{n-1}$ and $\operatorname{Char}(\Gamma)$ is generated by $\chi$ and $\chi_{i}=\operatorname{deg} x_{i}$ for $i \in$ $\{0, \ldots, n+1\}$ with relations

$$
\chi=5 \chi_{1}=3 \chi_{2}=2 \chi_{3}=\cdots=2 \chi_{n+1}=\chi_{0}+\cdots+\chi_{n+1} .
$$

5.6.1 If we write an element of $\operatorname{Jac}_{\mathbf{w}_{\gamma}} \otimes \Lambda^{\operatorname{dim} N_{\gamma}} N_{\gamma}^{\vee}$ as $x_{0}^{k_{0}} x_{1}^{k_{1}} x_{2}^{k_{2}} \otimes x_{j_{1}}^{\vee} \wedge x_{j_{2}}^{\vee} \wedge \cdots \wedge x_{j_{s}}^{\vee}$, then its degree is given by

$$
k_{0} \chi_{0}+k_{1} \chi_{1}+k_{2} \chi_{2}-\chi_{j_{1}}-\cdots-\chi_{j_{s}}
$$

which can be proportional to $\chi$ only if $V \cap\left(\mathbb{C} x_{3} \oplus \cdots \oplus \mathbb{C} x_{n+1}\right)$ is either $\mathbb{C} x_{3} \oplus \cdots \oplus \mathbb{C} x_{n+1}$ or 0 . We assume this condition for the rest of Section 5.6.

5.6.2 Since $t_{0}=1$ implies $t_{1}=t_{2}=1$, one has $\mathbb{C} x_{0} \subset V_{\gamma}$ if and only if either $V_{\gamma}=V$ or $V_{\gamma}=\mathbb{C} x_{0} \oplus \mathbb{C} x_{1} \oplus \mathbb{C} x_{2}$.

5.6.3 One has $V_{\gamma}=V$ if and only if $\gamma$ is the identity element. The degree of $x_{0}^{k_{0}} x_{1}^{k_{1}} x_{2}^{k_{2}} \in$ $\mathrm{Jac}_{\mathbf{w}}$ is

$$
k_{0} \chi-\left(k_{0}-k_{1}\right) \chi_{1}-\left(k_{0}-k_{2}\right) \chi_{2}-k_{0} \chi_{3}-\cdots-k_{0} \chi_{n+1},
$$

which is proportional to $\chi$ if and only if

-5 divides $k_{0}-k_{1}$ and 3 divides $k_{0}-k_{2}$ if $n=1$, and

- 5 divides $k_{0}-k_{1}, 3$ divides $k_{0}-k_{2}$, and $k_{0}$ is even if $n>1$. 


\section{SiMPLE SINGULARITIES}

For $n=1$, we must have

$$
7 k_{0}+3 k_{1}+5 k_{2}=15 m
$$

for $m \in \mathbb{N}$, in which case one has

$$
t:=2 \operatorname{deg}\left(x_{0}^{k_{0}} x_{1}^{k_{1}} x_{2}^{k_{2}}\right) / \chi=2 m .
$$

For each $m \in \mathbb{N}$ such that $7 \nmid m$, the equation (5.17) has a unique solution with $\left(k_{1}, k_{2}\right) \in$ $\{0,1,2,3\} \times\{0,1\}$, and if $7 \mid m$, then there are precisely two contributions with $\left(k_{1}, k_{2}\right)=(0,0)$ and $\left(k_{1}, k_{2}\right)=(3,1)$ such that $\left(k_{1}, k_{2}, m\right) \in\{0,1,2,3\} \times\{0,1\} \times \mathbb{N}$ except if $m=0$, in which case only $\left(k_{1}, k_{2}\right)=(0,0)$ contributes.

For $n>1$, we must have

$$
7 k_{0}+3 k_{1}+5 k_{2}=15 m
$$

for $m \in \mathbb{N}$, and in addition $k_{0}$ must be in $2 \mathbb{N}$. Thus, we can rewrite (5.18) as

$$
k_{0}=6 k_{1}+10 k_{2}+30 m^{\prime}
$$

with $m^{\prime}=k_{0} / 2-m$. One has

$$
t:=2 \operatorname{deg}\left(x_{0}^{k_{0}} x_{1}^{k_{1}} x_{2}^{k_{2}}\right) / \chi=12 k_{1}+20 k_{2}+58 m^{\prime}-\left(6 k_{1}+10 k_{2}+30 m^{\prime}\right) n .
$$

Each $\left(k_{1}, k_{2}, m^{\prime}\right) \in\{0,1,2,3\} \times\{0,1\} \times \mathbb{N}$ contributes $\mathbb{C}\left(k_{0} n\right)$ to $\mathrm{HH}^{t}$ and $\mathrm{HH}^{t+1}$.

5.6.4 If $n>1$, in addition, one has $V_{\gamma}=\mathbb{C} x_{0} \oplus \mathbb{C} x_{1} \oplus \mathbb{C} x_{2}$ if and only if $n$ is odd and $\gamma=(1,1,1,-1, \ldots,-1)$. The degree of $x_{0}^{k_{0}} x_{1}^{k_{1}} x_{2}^{k_{2}} \otimes x_{3}^{\vee} \wedge \cdots \wedge x_{n+1}^{\vee} \in \mathrm{Jac}_{\mathbf{w}_{\gamma}} \otimes \Lambda^{\operatorname{dim} N_{\gamma}} N_{\gamma}^{\vee}$ is

$$
k_{0} \chi-\left(k_{0}-k_{1}\right) \chi_{1}-\left(k_{0}-k_{2}\right) \chi_{2}-\left(k_{0}+1\right) \chi_{3}-\cdots-\left(k_{0}+1\right) \chi_{n+1},
$$

which is proportional to $\chi$ if and only if

$$
14 k_{0}+6 k_{1}+10 k_{2}=30 m
$$

for $m \in \mathbb{Z}$, and in addition we must have $k_{0}$ odd. Thus, again we can rewrite (5.19) as

$$
k_{0}=15+6 k_{1}+10 k_{2}+30 m^{\prime},
$$

where $m^{\prime}=\left(k_{0}-1\right) / 2-m$. One has

$$
\begin{aligned}
t & :=2 \operatorname{deg}\left(x_{0}^{k_{0}} x_{1}^{k_{1}} x_{2}^{k_{2}} \otimes x_{3}^{\vee} \wedge \cdots \wedge x_{n+1}^{\vee}\right) / \chi+\operatorname{dim} N_{\gamma} \\
& =2\left(k_{0}-\frac{1}{5}\left(k_{0}-k_{1}\right)-\frac{1}{3}\left(k_{0}-k_{2}\right)-\frac{1}{2}\left(k_{0}+1\right)(n-1)\right)+(n-1) \\
& =29+12 k_{1}+20 k_{2}+58 m^{\prime}-\left(15+6 k_{1}+10 k_{2}+30 m^{\prime}\right) n .
\end{aligned}
$$

Each $\left(k_{1}, k_{2}, m^{\prime}\right) \in\{0,1,2,3\} \times\{0,1\} \times \mathbb{Z}$ such that $15+6 k_{1}+10 k_{2}+30 m^{\prime} \geqslant 0$ contributes $\mathbb{C}\left(k_{0} n\right)$ to $\mathrm{HH}^{t}$ and $\mathrm{HH}^{t+1}$.

5.6.5 An element $\gamma=\left(t_{0}, \ldots, t_{n+1}\right) \in \operatorname{ker} \chi$ satisfies $V_{\gamma}=0$ if and only if $t_{1} \in \boldsymbol{\mu}_{5} \backslash\{1\}$, $t_{2} \in \boldsymbol{\mu}_{3} \backslash\{1\}, t_{3}=\cdots=t_{n+1}=-1$, and $t_{0}=(-1)^{n-1}\left(t_{1} t_{2}\right)^{-1}$. There are eight such elements, each of which contributes $\mathbb{C}(-n)$ to $\mathrm{HH}^{n}$.

\section{ACKNowledgements}

We thank the anonymous referees for reading the manuscript carefully and suggesting many improvements and corrections. 


\section{Y. LEKILI AND K. UEDA}

\section{REFERENCES}

AAK16 M. Abouzaid, D. Auroux, and L. Katzarkov, Lagrangian fibrations on blowups of toric varieties and mirror symmetry for hypersurfaces, Publ. Math. Inst. Hautes Études Sci. 123 (2016), 199-282; doi:10.1007/s10240-016-0081-9.

Abo11 M. Abouzaid, A topological model for the Fukaya categories of plumbings, J. Differential Geom. 87 (2011), no. 1, 1-80; doi:10.4310/jdg/1303219772.

ACH19 D. Arinkin, A. Căldăraru, and M. Hablicsek, Formality of derived intersections and the orbifold HKR isomorphism, J. Algebra 540 (2019), 100-120; doi:10.1016/ j.jalgebra.2019.08.002.

AGV85 V.I. Arnol'd, S. M. Guseřn-Zade, and A. N. Varchenko, Singularities of differentiable maps, Vol. I. The classification of critical points, caustics and wave fronts, Monogr. Math., vol. 82 (Birkhäuser Boston, Inc., Boston, MA, 1985); doi :10.1007/978-1-4612-5154-5.

AK19 M.A. Armenta and B. Keller, Derived invariance of the Tamarkin-Tsygan calculus of an algebra, C. R. Math. Acad. Sci. Paris 357 (2019), no. 3, 236-240; doi:10.1016/ j.crma.2019.01.007.

AR87 M. Auslander and I. Reiten, Almost split sequences for Z-graded rings, Singularities, Representation of Algebras, and Vector Bundles (Lambrecht, 1985), Lecture Notes in Math., vol. 1273 (Springer, Berlin, 1987), 232-243; doi:10.1007/BFb0078847.

Aur07 D. Auroux, Mirror symmetry and T-duality in the complement of an anticanonical divisor, J. Gökova Geom. Topol. GGT 1 (2007), 51-91.

Aur09 _ Special Lagrangian fibrations, wall-crossing, and mirror symmetry, in Surveys in Differential Geometry, Vol. XIII, Geometry, Analysis, and Algebraic Geometry: Forty Years of the Journal of Differential Geometry, Surv. Differ. Geom., vol. 13 (Int. Press, Somerville, MA, 2009), 1-47; doi:10.4310/SDG.2008.v13.n1.a1.

BEE12 F. Bourgeois, T. Ekholm, and Y. Eliashberg, Effect of Legendrian surgery (with an appendix by Sheel Ganatra and Maksim Maydanskiy), Geom. Topol. 16 (2012), no. 1, 301-389; doi: 10.2140/gt.2012.16.301.

BFK14 M. Ballard, D. Favero, and L. Katzarkov, A category of kernels for equivariant factorizations and its implications for Hodge theory, Publ. Math. Inst. Hautes Études Sci. 120 (2014), 1-111; doi:10.1007/s10240-013-0059-9.

BH93 P. Berglund and T. Hübsch, A generalized construction of mirror manifolds, Nuclear Phys. B 393 (1993), no. 1-2, 377-391; doi:10.1016/0550-3213(93) 90250-S.

BH95 P. Berglund and M. Henningson, Landau-Ginzburg orbifolds, mirror symmetry and the elliptic genus, Nuclear Phys. B 433 (1995), no. 2, 311-332; doi:10.1016/0550-3213(94)00389-V.

CDGG17 B. Chantraine, G. Dimitroglou Rizell, P. Ghiggini, and R. Golovko, Geometric generation of the wrapped Fukaya category of Weinstein manifolds and sectors, 2017, arXiv:1712.09126.

CJY04 R. L. Cohen, J. D. S. Jones, and J. Yan, The loop homology algebra of spheres and projective spaces, Categorical Decomposition Techniques in Algebraic Topology (Isle of Skye, 2001), Progr. Math., vol. 215 (Birkhäuser, Basel, 2004), 77-92; doi:10.1007/978-3-0348-78630_5.

CT13 A. Căldăraru and J. Tu, Curved $A_{\infty}$ algebras and Landau-Ginzburg models, New York J. Math. 19 (2013), 305-342.

Dyc11 T. Dyckerhoff, Compact generators in categories of matrix factorizations, Duke Math. J. 159 (2011), no. 2, 223-274; doi:10.1215/00127094-1415869.

EE07 P. Etingof and C.-H. Eu, Hochschild and cyclic homology of preprojective algebras of ADE quivers, Mosc. Math. J. 7 (2007), no. 4, 601-612; doi:10.17323/1609-4514-2007-7-4-601612.

Ekh19 T. Ekholm, Holomorphic curves for Legendrian surgery, 2019, arXiv:1906.07228. 


\section{SiMPLE SINGULARITIES}

EL17a T. Ekholm and Y. Lekili, Duality between Lagrangian and Legendrian invariants, 2017, arXiv: 1701.01284.

EL17b T. Etgü and Y. Lekili, Koszul duality patterns in Floer theory, Geom. Topol. 21 (2017), no. 6, 3313-3389; doi:10.2140/gt.2017.21.3313.

EL19 Fukaya categories of plumbings and multiplicative preprojective algebras, Quantum Topol. 10 (2019), no. 4, 777-813; doi:10.4171/qt/131.

EP15 A.I. Efimov and L. Positselski, Coherent analogues of matrix factorizations and relative singularity categories, Algebra Number Theory 9 (2015), no. 5, 1159-1292; doi:10.2140/ ant.2015.9.1159.

ES98a K. Erdmann and N. Snashall, On Hochschild cohomology of preprojective algebras. I, J. Algebra 205 (1998), no. 2, 391-412; doi:10.1006/jabr.1998.7547.

ES98b - On Hochschild cohomology of preprojective algebras. II, J. Algebra 205 (1998), no. 2, 413-434; doi:10.1006/jabr.1997.7329.

ES98c - Preprojective algebras of Dynkin type, periodicity and the second Hochschild cohomology, Algebras and Modules, II (Geiranger, 1996), CMS Conf. Proc., vol. 24 (Amer. Math. Soc., Providence, RI, 1998), 183-193.

Eu10 C.-H. Eu, The calculus structure of the Hochschild homology/cohomology of preprojective algebras of Dynkin quivers, J. Pure Appl. Algebra 214 (2010), no. 1, 28-46; doi:10.1016/ j.jpaa.2009.04.018.

FMT05 Y. Félix, L. Menichi, and J.-C. Thomas, Gerstenhaber duality in Hochschild cohomology, J. Pure Appl. Algebra 199 (2005), no. 1-3, 43-59; doi:10.1016/j.jpaa.2004.11.004.

FU11 M. Futaki and K. Ueda, Homological mirror symmetry for Brieskorn-Pham singularities, Selecta Math. (N.S.) 17 (2011), no. 2, 435-452; doi:10.1007/s00029-010-0055-6.

FU13 _ Homological mirror symmetry for singularities of type D, Math. Z. 273 (2013), no. 34, 633-652; doi:10.1007/s00209-012-1024-x.

Gan12 S. Ganatra, Symplectic cohomology and duality for the wrapped Fukaya category, Ph.D. Thesis, Massachusetts Institute of Technology, 2012, available at https://dspace.mit.edu/ handle/1721.1/73362.

Gao17 Y. Gao, Functors of wrapped Fukaya categories from Lagrangian correspondences, 2017, arXiv:1712.00225.

GDT90 I. M. Gel'fand, Yu. L. Daletskiü, and B. L. Tsygan, On a variant of noncommutative differential geometry, Soviet Math. Dokl. 40 (1990), no. 2, 422-426.

Gin06 V. Ginzburg, Calabi-Yau algebras, 2006, arXiv:math.AG/0612139.

Hab20 M. Habermann, Homological mirror symmetry for invertible polynomials in two variables, 2020, arXiv: 2003.01106.

Her19 E. Herscovich, Hochschild (co)homology of Koszul dual pairs, J. Noncommut. Geom. 13 (2019), no. 1, 59-85; doi:10.4171/JNCG/323.

HS20 M. Habermann and J. Smith, Homological Berglund-Hübsch mirror symmetry for curve singularities, J. Symplectic Geom. 18 (2020), no. 6, 1515-1574; doi:10.4310/JSG.2020.v18.n6.a2.

IT13 O. Iyama and R. Takahashi, Tilting and cluster tilting for quotient singularities, Math. Ann. 356 (2013), no. 3, 1065-1105; doi:10.1007/s00208-012-0842-9.

Kel11 B. Keller, Deformed Calabi-Yau completions (with an appendix by Michel Van den Bergh), J. reine angew. Math. 654 (2011), 125-180; doi:10.1515/CRELLE.2011.031.

KMVdB11 B. Keller, D. Murfet, and M. Van den Bergh, On two examples by Iyama and Yoshino, Compos. Math. 147 (2011), no. 2, 591-612; doi:10.1112/S0010437X10004902.

Knö87 H. Knörrer, Cohen-Macaulay modules on hypersurface singularities. I, Invent. Math. 88 (1987), no. 1, 153-164; doi:10.1007/BF01405095. 


\section{Y. LEKILI AND K. UEDA}

KST07 H. Kajiura, K. Saito, and A. Takahashi, Matrix factorization and representations of quivers. II. Type ADE case, Adv. Math. 211 (2007), no. 1, 327-362; doi:10.1016/ j.aim.2006.08.005.

KvK16 M. Kwon and O. van Koert, Brieskorn manifolds in contact topology, Bull. Lond. Math. Soc. 48 (2016), no. 2, 173-241; doi:10.1112/blms/bdv088.

Li19 Y. Li, Koszul duality via suspending Lefschetz fibrations, J. Topol. 12 (2019), no. 4, 11741245; doi:10.1112/topo.12113.

LU18 Y. Lekili and K. Ueda, Homological mirror symmetry for Milnor fibers via moduli of $A_{\infty}{ }^{-}$ structures, 2018, arXiv:1806.04345.

Orl11 D. Orlov, Formal completions and idempotent completions of triangulated categories of singularities, Adv. Math. 226 (2011), no. 1, 206-217; doi:10.1016/j.aim.2010.06.016.

Seg13 E. Segal, The closed state space of affine Landau-Ginzburg B-models, J. Noncommut. Geom. 7 (2013), no. 3, 857-883; doi:10.4171/JNCG/137.

Sei01 P. Seidel, More about vanishing cycles and mutation, Symplectic Geometry and Mirror Symmetry (Seoul, 2000) (World Sci. Publ., River Edge, NJ, 2001), 429-465; doi:10.1142/ $9789812799821 \_0012$.

Sei10 Suspending Lefschetz fibrations, with an application to local mirror symmetry, Comm. Math. Phys. 297 (2010), no. 2, 515-528; doi:10.1007/s00220-009-0944-8.

Sei12 Lagrangian homology spheres in $\left(A_{m}\right)$ Milnor fibres via $\mathbb{C}^{*}$-equivariant $A_{\infty}$-modules, Geom. Topol. 16 (2012), no. 4, 2343-2389; doi:10.2140/gt.2012.16.2343.

SS12 P. Seidel and J. P. Solomon, Symplectic cohomology and q-intersection numbers, Geom. Funct. Anal. 22 (2012), no. 2, 443-477; doi:10.1007/s00039-012-0159-6.

Tak05 A. Takahashi, Matrix factorizations and representations of quivers I, 2005, arXiv:math.AG/ 0506347.

TT00 D. Tamarkin and B. Tsygan, Noncommutative differential calculus, homotopy BV algebras and formality conjectures, Methods Funct. Anal. Topology 6 (2000), no. 2, 85-100.

Ueb16 P. Uebele, Symplectic homology of some Brieskorn manifolds, Math. Z. 283 (2016), no. 1-2, 243-274; doi:10.1007/s00209-015-1596-3.

Vit18 C. Viterbo, Functors and computations in Floer homology with applications Part II, arXiv: 1805.01316.

Yankı Lekili y.lekili@imperial.ac.uk

Department of Mathematics, Imperial College London, South Kensington, London SW7 2AZ, UK

Kazushi Ueda kazushi@ms.u-tokyo.ac.jp

Graduate School of Mathematical Sciences, The University of Tokyo, 3-8-1 Komaba, Meguro-ku, Tokyo, 153-8914, Japan 